\title{
Studies of bacterial branching growth using reaction-diffusion models for colonial development
}

\author{
Ido Golding*, Yonathan Kozlovsky, Inon Cohen, Eshel Ben-Jacob \\ School of Physics and Astronomy, Raymond and Beverly Sackler Faculty of Exact Sciences, \\ Tel Aviv University, Tel Aviv 69 978, Israel
}

Received 28 June 1998

\begin{abstract}
Various bacterial strains exhibit colonial branching patterns during growth on poor substrates. These patterns reflect bacterial cooperative self-organization and cybernetic processes of communication, regulation and control employed during colonial development. One method of modeling is the continuous, or coupled reaction-diffusion approach, in which continuous time evolution equations describe the bacterial density and the concentration of the relevant chemical fields. In the context of branching growth, this idea has been pursued by a number of groups. We present an additional model which includes a lubrication fluid excreted by the bacteria. We also add fields of chemotactic agents to the other models. We then present a critique of this whole enterprise with focus on the models' potential for revealing new biological features. (C) 1998 Elsevier Science B.V. All rights reserved.
\end{abstract}

PACS: 47.20.Hw; 87.10.e

Keywords: Bacillus-subtilis; Reaction-diffusion; Morphology transition; Model; Organization

\section{Introduction}

During the course of evolution, bacteria have developed sophisticated cooperative behavior and intricate communication capabilities [1-5]. These include: direct cellcell physical interactions via extra-membrane polymers [6,7], collective production of extracellular "wetting" fluid for movement on hard surfaces [8,9], long range chemical signaling, such as quorum sensing [10-12] and chemotactic signaling ${ }^{1}[13-15]$,

\footnotetext{
* Corresponding author. E-mail: golding@orion.tau.ac.il.

${ }^{1}$ Chemotaxis is a bias of movement according to the gradient of a chemical agent. Chemotactic signaling is a chemotactic response to an agent emitted by the bacteria.
} 
collective activation and deactivation of genes [16-18] and even exchange of genetic material [19-21]. Utilizing these capabilities, bacterial colonies develop complex spatio-temporal patterns in response to adverse growth conditions.

It is now understood that the study of cooperative self-organization of bacterial colonies is an exciting new multidisciplinary field of research, necessitating the merger of biological information with the physics of non-equilibrium processes and the mathematics of non-linear dynamics. At this stage, several experimental systems have been identified, and preliminary modeling efforts are making significant progress in providing a framework for the understanding of experimental observations [8,18,22-32].

Fujikawa and Matsushita $[23,33,34]$ reported for the first time ${ }^{2}$ that bacterial colonies could grow elaborate branching patterns of the type known from the study of fractal formation in the process of diffusion-limited-aggregation (DLA) [37-39]. This work was done with Bacillus subtilis, but was subsequently extended to other bacterial species such as Serratia marcescens and Salmonella anatum [40]. It was shown explicitly that nutrient diffusion was the relevant dynamics responsible for the growth instability. Later, we will see how models which couple nutrient diffusion to bacterial density can naturally account for these structures.

Motivated by these observations, Ben-Jacob et al. [25,27,41] conducted new experiments to see how adaptive bacterial colonies could be in the presence of external "pressure", here in the form of a limited nutrient supply and hard surface. The endeavor started with $B$. subtilis 168 , which is non-motile on a solid agar surface, from which a new species of bacteria has been isolated $[25,41]$. The new species was designated Paenibacillus dendritiformis var. dendron [42]. This species is motile on the hard surface and its colonies exhibit branching patterns (Fig. 1). The new mode of tipsplitting growth was found to be inheritable and transferable by a single cell, hence it is referred to as a distinctive morphotype [43], and, to indicate the tip-splitting character of the growth, it was denoted $\mathscr{T}$ morphotype. In the next section we describe in some detail the observations of Ben-Jacob et al. and Matsushita et al. Additional studies of branching colonial growth are reported by Matsuyama et al. $[8,44]$ and Mendelson and Salhi [18].

All the various strains reported in the studies quoted above exhibit branching patterns during growth on a poor substrate. Drawing on the analogy with diffusive patterning in non-living systems [45-48], we can state that complex patterns are expected. The cellular reproduction rate that determines the growth rate of the colony is limited by the level of nutrients available for the cells. The latter is limited by the diffusion of nutrients towards the colony (for low nutrient substrate). Hence colony growth under certain conditions should be similar to diffusion limited growth in non-living systems as mentioned above [47,48]. The study of diffusive patterning in non-living systems teaches us that the diffusion field drives the system towards decorated (on many length scales) irregular fractal shapes. Indeed, bacterial colonies can develop patterns

\footnotetext{
${ }^{2}$ We refer to the first time that branching growth was studied as such. Observations of branching colonies occurred long ago $[35,36]$.
} 


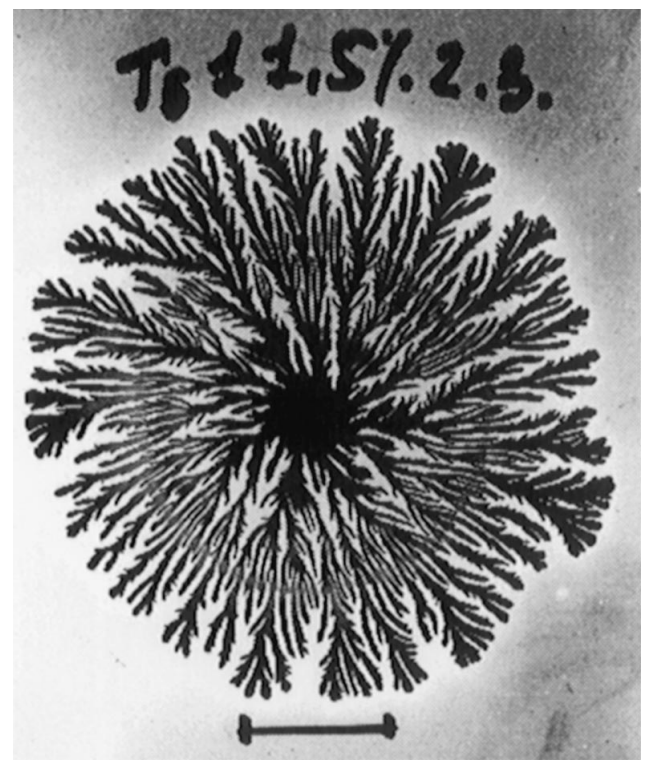

Fig. 1. Typical example of branching growth of the $\mathscr{T}$ morphotype for $1 \mathrm{~g} / 1$ peptone level and $1.5 \%$ agar concentration.

reminiscent of those observed during growth in non-living systems. But, this is certainly not the end of the story. The colonies exhibit a richer behavior. This, ultimately, is a reflection of the additional levels of complexity involved when the building blocks of the colonies, the bacteria, are themselves living systems. We now start to reveal the cybernetic processes (communication, regulation and control) which are part of the colonial adaptive self-organization, and their determination of the interaction between genetic information and biophysical behavior.

How should one approach the modeling of the complex bacterial patterning? With present computational power it is natural to use computer models as a main tool in the study of complex systems. However, one must be careful not to be trapped in the "reminiscence syndrome", described by Cowan [49], as the tendency to devise a set of rules which will mimic some aspect of the observed phenomena and then, to quote J.D. Cowan “They say: 'Look, isn't this reminiscent of a biological or physical phenomenon!' They jump in right away as if it's a decent model for the phenomenon, and usually of course it's just got some accidental features that make it look like something". Yet the reminiscence modeling approach has some indirect value. True, doing so does not reveal (directly) the biological functions and behavior. However, it does reflect understanding of geometrical and temporal features of the patterns, which indirectly might help in revealing the underlying biological principles. Another extreme is the "realistic modeling" approach, where one constructs an algorithm that includes in details all the known biological facts about the system. Such an approach sets 
a trajectory of ever including more and more details (vs. generalized features). The model keeps evolving to include so many details that it loses any predictive power.

Here we try to promote another approach - the "generic modeling" one [50,27,51,31]. We seek to elicit, from the experimental observations and the biological knowledge, the generic features and basic principles needed to explain the biological behavior and to include these features in the model. We will demonstrate that such modeling, with close comparison to experimental observations, can be used as a research tool to reveal new understanding of the biological systems.

Generic modeling is not about using sophisticated, as it may, mathematical description to dress pre-existing understanding of complex biological behavior. Rather, it means a cooperative approach, using existing biological knowledge together with mathematical tools and synergetic point of view for complex systems to reach a new understanding (which is reflected in the constructed model) of the observed complex phenomena.

The generic models can yet be grouped into two main categories: (1) Discrete models such as the communicating walkers models of Ben-Jacob et al. [27,52,30] and the bions model of Kessler and Levine [50,53]. In this approach, the microorganisms (bacteria in the first model and amoebae in second) are represented by discrete, random walking entities (walkers and bions, respectively) which can consume nutrients, reproduce, perform random or biased movement, and produce or respond to chemicals. The time evolution of the chemicals is described by reaction-diffusion equations. (2) Continuous or reaction-diffusion models [54,55]. In these models the microorganisms are represented via their $2 \mathrm{D}$ density, and a reaction-diffusion equation of this density describes their time evolution. This equation is coupled to the other reactiondiffusion equations for the chemical fields. In the context of branching growth, this idea has been pursued recently by Mimura and Matsushita et al. [56,57], Kawasaki et al. [58] and Kitsunezaki [59]. A summary and critique of this approach is provided by Rafols [60].

Here we describe a new model which includes a lubrication fluid and a model with a cutoff, as was proposed by Kessler and Levine [61]. We compare the results obtained by the various models and the experimental observations. Our main goal is to identify the biological and mathematical requirements for branching patterns. We then study the effect of nutrients- and signaling-chemotaxis, and conclude that chemotaxis is needed to explain the wealth of experimental observations.

\section{Experimental results}

Several strains of bacteria were reported to produce tip-splitting branched patterns under conditions of low level of nutrient. We describe here the experimental results of Ben-Jacob et al. $[25,41,27,62]$ - working with colonies of Paenibacillus dendritiformis var. dendron ( $\mathscr{T}$ morphotype) - and Matsushita, Fujikawa, Matsuyama and coworkers $[33,23,34,26,40]$ - working with colonies of Bacillus subtilis. 


\subsection{Growth patterns of $\mathscr{T}$ morphotype}

(a) Macroscopic observations. All manner of patterns are exhibited by $\mathscr{T}$ morphotype as the growth conditions are varied. An example of branching pattern is shown in Fig. 1. This kaleidoscope of shapes may be grouped into a number of "essential" patterns. For intermediate agar concentrations (about $1.5 \%-1.5 \mathrm{~g}$ in $100 \mathrm{ml}$ ), at very high peptone levels (above $10 \mathrm{~g} / \mathrm{l}$ ) the patterns are compact (Fig. 2a). At somewhat lower but still high peptone levels (about 5-10 g/1) the patterns, reminiscent of viscous fingering patterns in Hele-Shaw devices [47], exhibit quite pronounced radial symmetry and may be characterized as dense fingers (Fig. 2b), each finger being much wider than the distance between fingers. For intermediate peptone levels, branching patterns with lower fractal dimension (reminiscent of electro-chemical deposition) are observed (Fig. 2c). The patterns are "bushy", with branch width smaller than the distance between branches. As the peptone level is lowered, the patterns become more ramified and fractal-like. Surprisingly, at even lower peptone levels (below $0.25 \mathrm{~g} / 1$ for $2 \%$ agar concentration) the colonies revert to organized structures: fine branches forming a well defined global envelope. We characterize these patterns as fine radial branches (Fig. 2d). For extremely low peptone levels (below $0.1 \mathrm{~g} / 1$ ), the colonies lose the fine radial structure and again exhibit fractal patterns (Fig. 3a). For high agar concentration the branches are very thin (Fig. 3b).

At high agar concentration and intermediate peptone levels the colonies display a structure of concentric rings superimposed on a branching pattern (Fig. 4a). At high agar concentration and very high peptone levels the colonies display a structure of concentric rings in a compact colony (Fig. 4b). At high agar concentrations the branches also exhibit a global twist with the same handedness, as shown in Fig. 4c. Similar observations during growth of other bacterial strains have been reported by Matsuyama et al. [26,44]. We referred to such growth patterns as having weak chirality $[3,52]$.

A closer look at an individual branch (Fig. 4d) reveals a phenomenon of density variations within the branches. These three-dimensional structures arise from accumulation of cells in layers. The aggregates can form spots and ridges which are either scattered randomly, ordered in rows, or organized in a leaf-veins-like structure. The aggregates are not frozen; the cells in them are motile and the aggregates are dynamically maintained.

At the other extreme, of very soft agar $(0.5 \%$ and below $)$, the $\mathscr{T}$ morphotype does not exhibit branching patterns. Instead, the growth is compact with density. In the range of $0.5-1 \%$ agar concentration the colonies typically have a shape of many arms stars.

(b) Microscopic observations. Under the microscope, bacterial cells are seen to perform a random-walk-like movement in a layer of fluid on the agar surface. This wetting fluid is assumed to be excreted by the cells and/or drawn by the cells from the agar $[27,62]$. The cellular movement is confined to this fluid; isolated cells spotted on the 

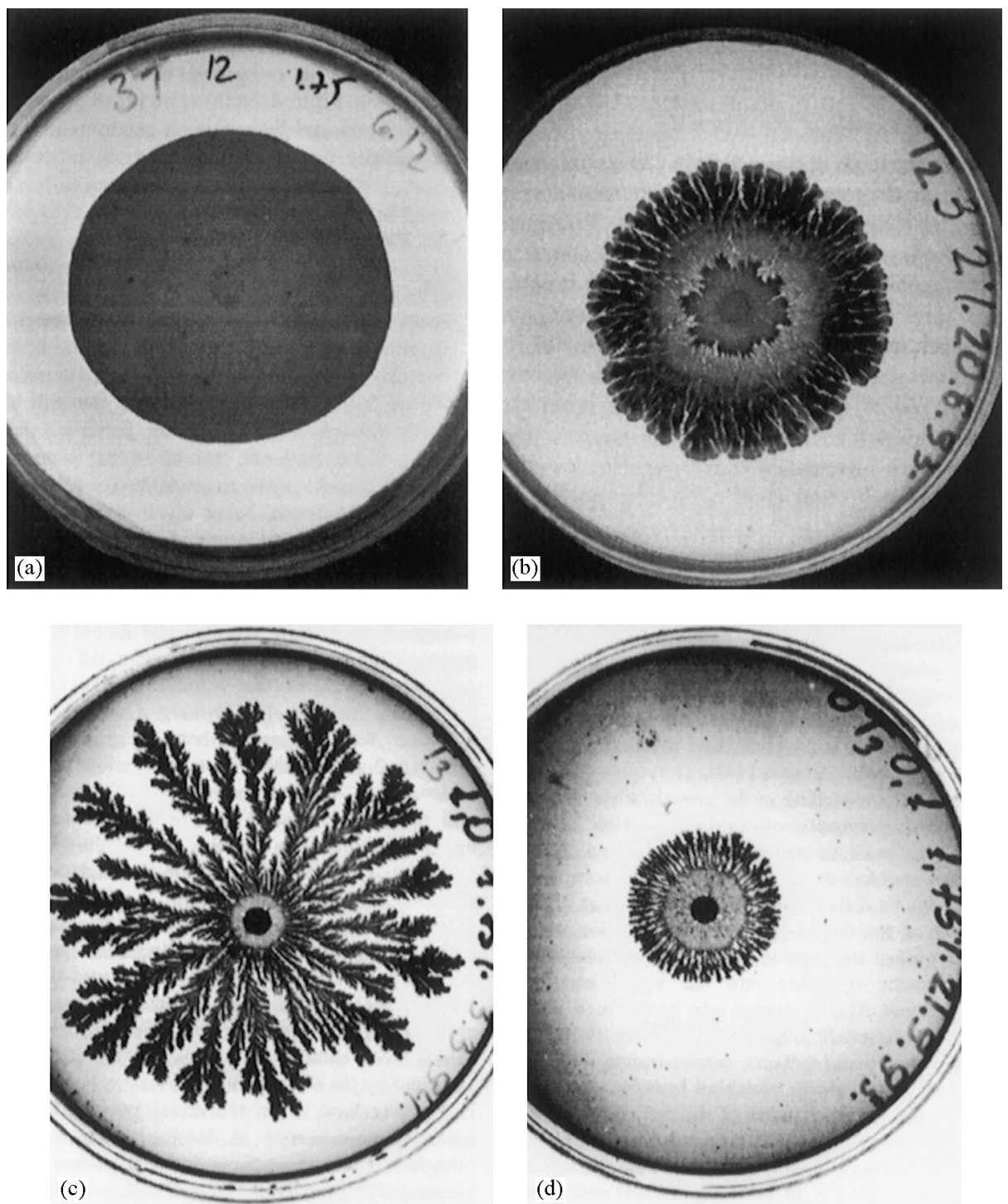

Fig. 2. Examples of typical patterns of $\mathscr{T}$ morphotype for intermediate agar concentration (clockwise from top left). (a) At very high peptone level (peptone $12 \mathrm{~g} / 1$, agar concentration $1.75 \%$ ) the pattern is compact. (b) At high peptone level $(3 \mathrm{~g} / 1$, agar $2 \%)$ the pattern is of dense fingers with pronounced radial symmetry - similar to patterns observed in Hele-Shaw cell. (c) At intermediate peptone level ( $1 \mathrm{~g} / 1$, agar $1.75 \%)$ the pattern is "bushy" fractal-like pattern, with branch width smaller than the distance between branches. (d) At low peptone level $(0.1 \mathrm{~g} / \mathrm{l}$, agar $1.75 \%)$ there are fine radial branches with apparent circular envelope.

agar surface do not move. This is an important observation as we discuss later when formulating the models. The fluid's boundary thus defines a local boundary for the branch. Whenever the cells are active, the boundary propagates slowly, as a result of the cellular movement and production of wetting fluid. 

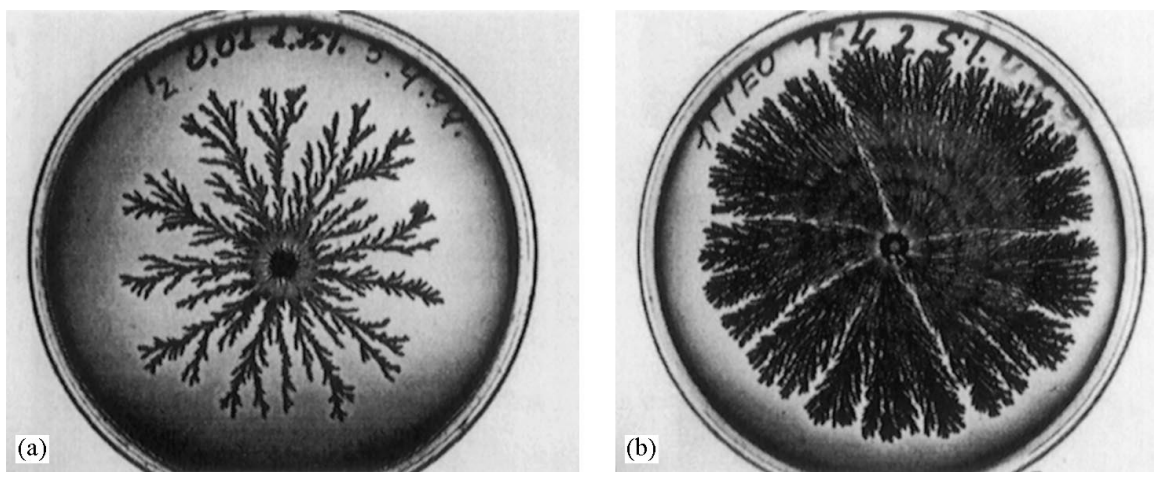

Fig. 3. (left) Fractal pattern for $0.01 \mathrm{~g} / 1$ peptone level and $1.75 \%$ agar concentration. (right) Dense branching pattern for $4 \mathrm{~g} / 1$ peptone and $2.5 \%$ agar. Note that the branches are much thinner than those in Fig. $2 \mathrm{~b}$, i.e. the branches are thinner for higher agar concentrations.

At very low agar concentrations (below $0.5 \%$ ) the bacteria swim inside the agar and not on its surface. Between $0.5 \%$ and $1 \%$ agar concentration some of the bacteria move on the surface and some inside the agar.

The observations reveal also that the cells are active at the outer parts of the colony, while closer to the center the cells are stationary (do not move) and some of them sporulate (form spores). It is known that certain bacteria respond to adverse growth conditions by entering a spore stage until more favorable growth conditions return. Such spores are metabolically inert and exhibit a marked resistance to the lethal effects if heat, drying, freezing, deleterious chemicals, and radiation.

\subsection{Morphology selection, morphology diagram and velocity-pattern correlations}

The emerging understanding of pattern determination in non-living includes the concepts of morphology diagram, morphology selection, morphology velocity correlations and morphology transitions [48]. In short, the patterns formed in many evolving azoic (non-living) systems may often be grouped into a small number of "essential shapes" or morphologies each representing a dominance of a different underlying effect. If each morphology is observed over a range of growth conditions, a morphology diagram may exist. The existence of a morphology diagram implies the existence of a morphology selection principle and vice versa. Ben-Jacob et al. proposed the existence of a new morphology selection principle: the principle of the fastest growing morphology $[63,47]$, a principle which should be applicable for a wide range of growth conditions. In general, if more than one morphology is a possible solution, only the fastest growing one is nonlinearly stable and will be observed, that is, selected.

The new selection principle implies that the average velocity is an appropriate response function for describing the growth processes and hence should be correlated with the geometrical character of the growth. In other words, for each regime (essential shape) in the morphology diagram, there is a characteristic functional dependence 

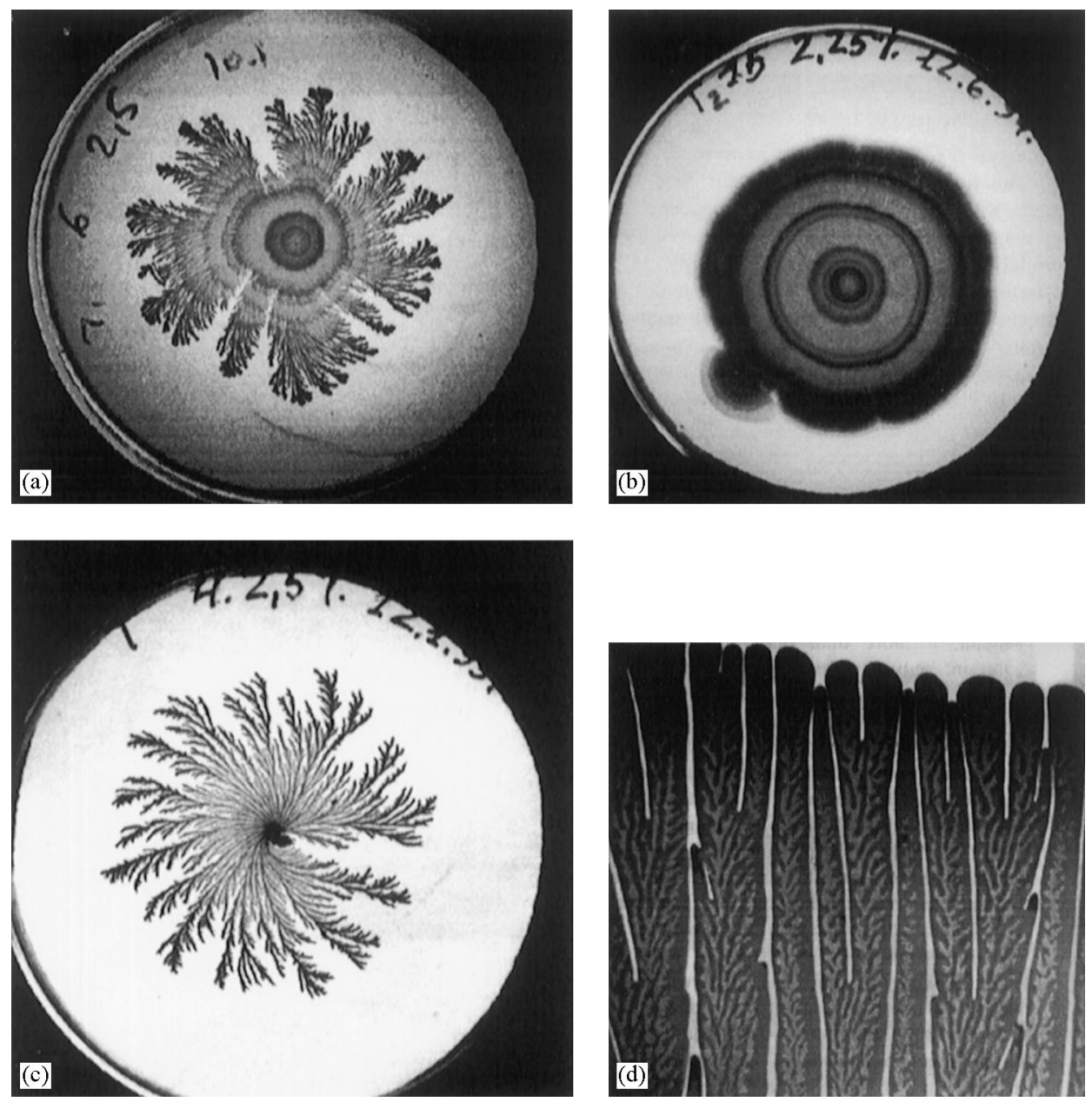

Fig. 4. Patterns of colonies of $\mathscr{T}$ morphotype (clockwise from top left). (a) Pattern of concentric rings superimposed on a branched colony for $2.5 \mathrm{~g} / 1$ peptone level and $2.5 \%$ agar concentration. (b) Concentric rings in a compact growth for $15 \mathrm{~g} / 1$ peptone level and $2.25 \%$ agar concentration. (c) Weak chirality (global twist of the branches) for $4 \mathrm{~g} / 1$ peptone and $2.5 \%$ agar concentration. (d) Closer look at the branches $(\times 50$ magnification) shows density variations within each branch. Darker colors represent thicker layer of bacteria.

of the velocity on the growth parameters. At the boundaries between the regimes there is either discontinuity in the velocity (first order-like transition) or in its slope (second order-like transition).

At present, there is some evidence for the existence of the new selection principle in non-living systems. The new principle might also be valid for pattern determination during colonial development in bacteria $[3,41]$. The bacterial patterns may be grouped into a small number of "essential shapes", each observed over a range of growth 
conditions $[23,34,25,41,18]$. To prove this hypothesis, the next step would be to demonstrate the velocity-pattern correlation during colonial growth.

A plot of the growth velocity as a function of nutrient level for $1.5 \%$ agar concentration is shown in Ref. [62]. For the presented range of peptone levels it was found that the velocity shows three distinct regimes of response, each corresponding also to a distinct morphology (the fine radial branches, branching patterns and dense fingers), as was predicted for non-living systems. The change in velocity suggests that the switching between morphologies is indeed a real morphology transition and not a simple cross-over (see Ref. [48]). The transition at low peptone level (between fine radial branches and branching structure) might be a first order morphology transition, i.e. a transition characterized by a jump in the velocity and hysteresis. The transition at the higher peptone level (from branches to dense fingering) seems to be second-order-like. These observations of velocity-pattern correlations strongly support the existence of a morphology selection principle which determines the selected colonial morphology for a given morphotype.

In non-evolving (equilibrium) systems there is a phenomenon of critical fluctuations when the system is kept at the transition point between two phases. At that point the system consists of a mixture of the two phases. In Ref. [48] it was shown that an analogous phenomenon exists in evolving non-living systems and explained that this fact provides additional support for the idea of morphology transitions. Fig. 5 shows patterns exhibited by colonies grown at "critical" peptone levels, where transitions between two morphologies occur. Similarly, for the fluctuations displayed by non-living systems, we observe a combination of the morphologies characterizing the patterns above and below the critical point. These observations provide additional support for the relevance of the concepts of morphology selection and morphology transition to colonial development.

\subsection{Growth patterns of B. subtilis}

Matsushita and coworkers [33,23,34,26,40] studied the colonial branching patterns and morphology diagram of the bacteria specie $B$. subtilis OG-01. A detailed summary of these observations is provided by Rafols [60]. A typical morphology diagram is shown in Fig. 6. Note that here the $x$-axis is the inverse agar concentration and the $y$-axis is the nutrient level. These bacteria are not efficient in producing a lubricating fluid, hence above about $0.8 \%$ agar concentration they cannot move on the agar surface: Under such conditions and low level of nutrients (below $1 \mathrm{~g} / 1$ peptone), DLAlike patterns are observed. As the level of nutrients is increased, the patterns become compact, with a cellular structure at the interface.

For low agar concentrations (below $0.5 \%$, so that the bacteria can move) and low level of nutrients, dense branching patterns are observed. These patterns are replaced by compact growth for higher levels of nutrients. Beautiful patterns of concentric rings imposed on a dense branching growth (Fig. 7) are observed at high levels of nutrients and intermediate agar concentric (about $0.75 \%$ ). For more details see Ref. [60]. 


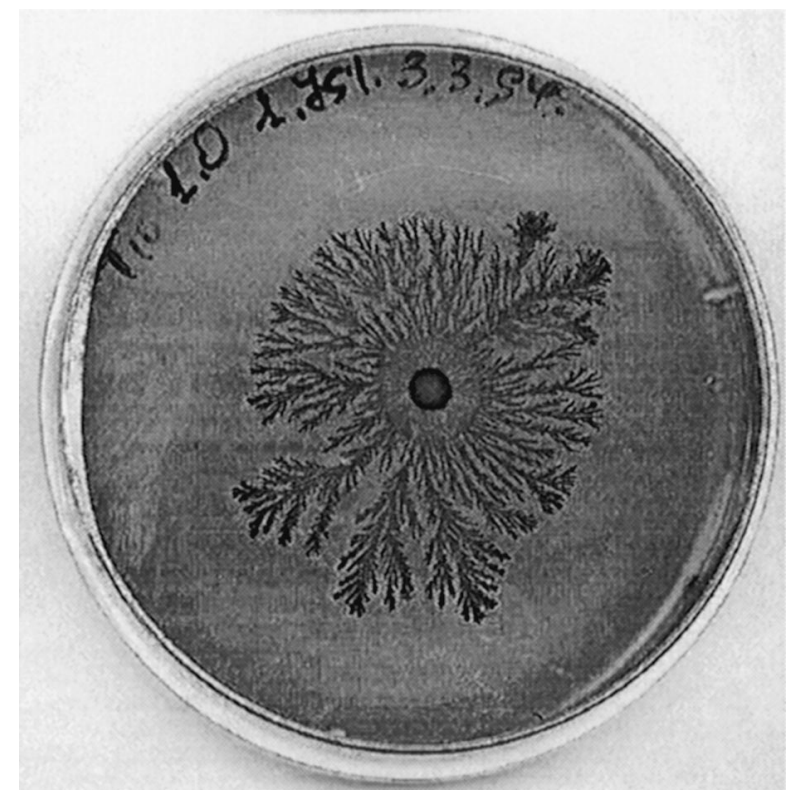

Fig. 5. Coexistence of two morphologies near the critical point. The colony shows combination of the radial symmetry morphology and the fractal-like morphology. The colony is grown on agar concentration of $1.75 \%$ and $1 \mathrm{~g} / 1$ peptone level.

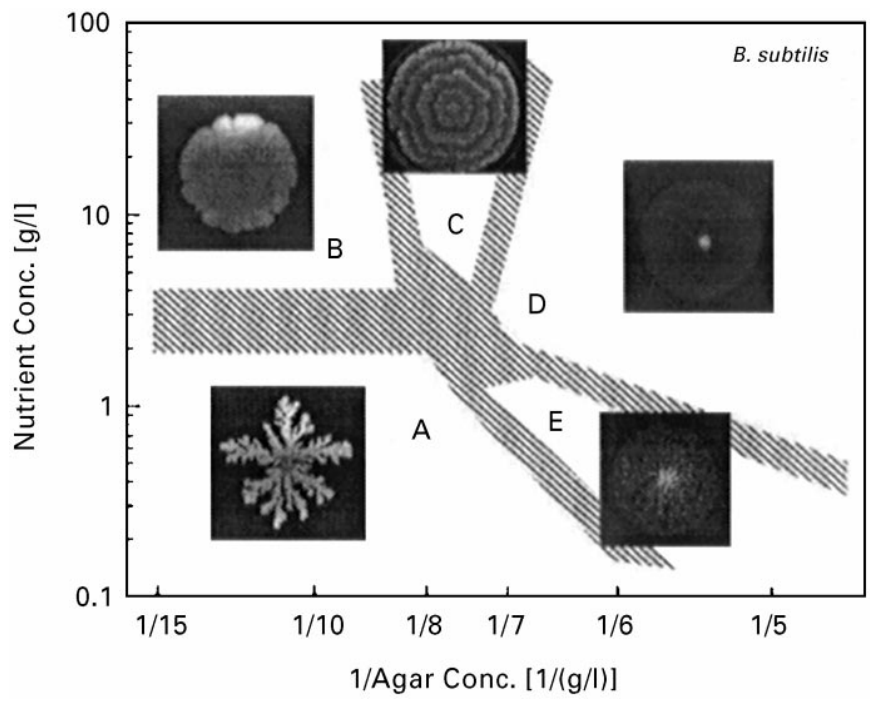

Fig. 6. Morphology diagram of $B$. subtilis colonies grown by Matsushita et al. $[23,33,26]$. Taken with permission from [60]. 


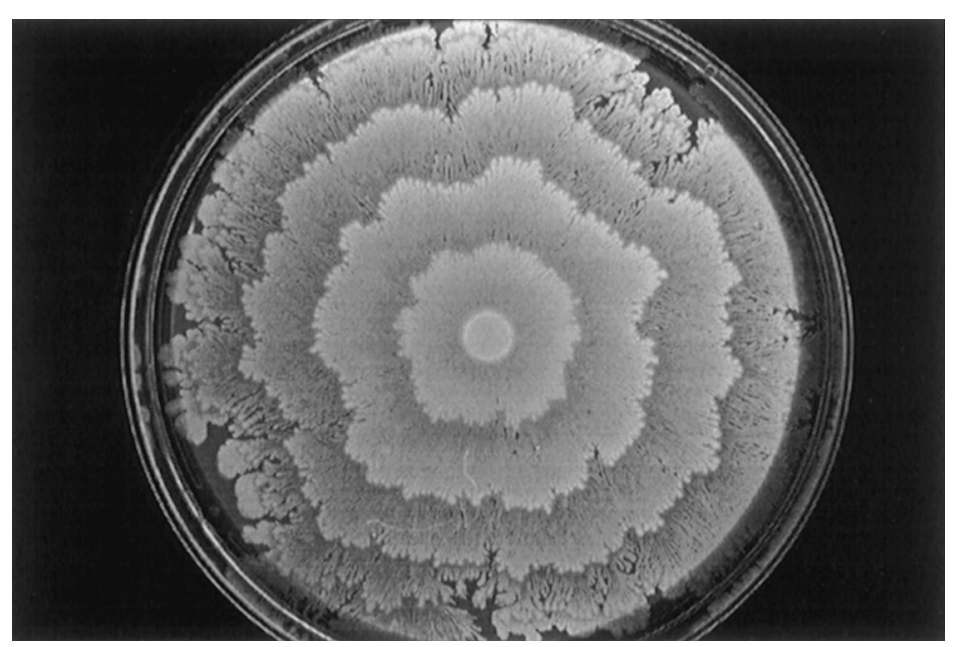

Fig. 7. Colony of B. subtilis. Pattern of concentric rings superimposed on a branched colony. Taken with permission from [60].

The different morphologies correspond to different growth velocities; DLA-like patterns grow in about a month, compact patterns at intermediate concentrations of agar grow in about a week, dense branching patterns and patterns of concentric rings grow in few days, and compact patterns at low concentrations of agar grow in half a day. From this we learn that indeed the growth velocity of the various morphologies is very different. Moreover, it seems that different bacterial movement mechanisms correspond to the different regimes. Thus we expect a real transition between the various regimes in the morphology diagram rather than a simple cross-over. Therefore, if velocity as function of the growth parameters is to be measured, it will probably show a jump in the velocity or its slope (first or second morphology transitions, respectively).

\section{Biological background}

Clearly we cannot begin to encompass all the biological background. Thus we will describe here, based on our previous experience, only the most relevant information for the understanding and modeling of the observed colonial patterning.

\subsection{Bacterial surface translocation}

The most widely studied mechanism used by bacteria for movement is swimming with flagella [64], but other mechanisms exist as well [65]. Most common types of bacterial movements are categorized to be

- Swimming - Surface translocation produced through the action of flagella. The cells move individually and at random in the same manner as flagellated bacteria move 
in wet mounts (i.e., nearly straight runs separated by brief tumbling). Swimming takes place only in sufficiently thick surface fluid. Microscope observations reveal no organized flow-field pattern.

- Swarming - Surface translocation produced through the action of flagella, but unlike swimming, the movement is continuous and regularly follows the long axis of the cell. The cells are predominantly aggregated in bundles during their movement, and microscope observations reveal flow-field patterns highly organized in whirls and bands.

- Gliding - Surface translocation occurring only in non-flagellated bacteria and only when in contact with solid surface. In all other respects, gliding is identical to swarming.

\subsection{Modeling bacterial movement}

As for the movement of $\mathscr{T}$ morphotype, based on microscope observations of movement and electron microscope observations of flagella we identify the movement as swimming. Cells tumble about every $\tau_{T} \approx 1-5$ depending on external conditions. The speed of the bacterium between tumbling events is very sensitive to conditions such as the liquid viscosity, temperature and $\mathrm{pH}$ level. Typically, it is of the order of $1-10 \mu \mathrm{m} / \mathrm{s}$.

Swimming can be approximated by a random walk with variable step size [66]. At low bacterial densities the random walk can be described by a diffusion equation with a diffusion coefficient $D_{b} \equiv v^{2} \tau_{T}=10^{-8}-10^{-5} \mathrm{~cm}^{2} / \mathrm{s}$. Low bacterial densities means that the mean free path between bacterial collisions $l_{c}$ is longer than the tumbling length $l_{T} \equiv v \tau_{T}$, thus collisions between the bacteria can be neglected. The mean free path (or collision length) is

$$
l_{c} \propto \begin{cases}\rho^{-1 / 3} & \text { in } 3 \text { dimensions }, \\ \sigma^{-1 / 2} & \text { in } 2 \text { dimensions },\end{cases}
$$

where $\rho$ is the 3D bacterial density and $\sigma$ is the $2 \mathrm{D}$ density - the projection of $\rho$ on the surface.

At high densities $\left(l_{c}<l_{T}\right)$, the collisions cannot be neglected. In attempt to approximate the dynamics in those conditions, one may want to consider the time of straight motion to be $l_{c} / v$ instead of $\tau_{T}$. Hence $D_{b}$ depends on the bacterial density to yield

$$
D_{b} \propto \begin{cases}v \rho^{-1 / 3} & \text { in } 3 \mathrm{D}, \\ v \sigma^{-1 / 2} & \text { in } 2 \mathrm{D} .\end{cases}
$$

This approximation is valid under the assumptions that a collision event is identical to a tumbling event (abrupt uncorrelated change in direction of motion), that a tumbling event is independent of the collisions, and that the speed between such events is not affected by their frequency.

The assumption that a collision event is like a tumbling event poses many problems. Even if the bacteria do not activate special response to collision it is unrealistic to assume that collisions are elastic, or that the flagella adopt immediately to the new 
orientation which changes during collisions. Thus it is reasonable to assume strong correlation between the cell's orientation before collision and the cell's orientation after collision. In addition, the orientation after the collision should be biased according with the average direction of motion of the surrounding bacteria, as they carry the liquid with them. The important parameter is not the collision length $l_{c}$ but re-orientation time $\tau_{r}$. The re-orientation time is the time it takes a bacterium to loose memory of its initial orientation, i.e. the time span on which the final orientation has effectively no correlation with the initial orientation. At low densities the re-orientation time $\tau_{r}$ is equal to the tumbling time $\tau_{T}$. As the density rises and the collisions become more frequent, $\tau_{r}$ decrease. $\tau_{r}$ defines the densities above which the constant diffusion coefficient $D_{b} \equiv v^{2} \tau_{T}$ is not a good approximation. It is quite possible that these densities are high enough so as to make the velocity and even the type of motion dependent on bacterial density, making relation (2) irrelevant. In any case, high cellular densities does mean an effective decrease in the diffusion coefficient related to the bacterial movement.

When swimming in an unstirred liquid, very low cellular densities also effect the movement. The bacteria secrete various materials into the media and some of them, e.g. enzymes and other polymers, change significantly the physical properties of the liquid making it more suitable for bacterial swimming. The secretion of these materials depend on cellular density, thus at not-too-high densities the speed of swimming rise with the cellular density. Hence the diffusion coefficient related to the bacterial movement should be a non-monotonic function of the bacterial density. Moreover, the specific functional form might depend on the specific bacterial strain.

In other conditions there is similar but more pronounced effect. On semi-solid surface the bacteria cannot swim at all inside the agar and they have to produce their own layer of liquid to swim in it. A single $\mathscr{T}$ bacterium on the agar surface cannot produce enough fluid to swim in it, thus the bacteria cannot break out of the layer fluid and the branches of a $\mathscr{T}$ colony can be defined by this fluid. Whenever bacteria enter the shallower parts of the layer, at the edge of the branch, they become sluggish, indicating that the depth of the layer effects the bacterial movement. It can be argued (see Section 4.4) that in such cases the bacterial speed is related to the bacterial density by a power law (at least in low densities). Not only the diffusion coefficient related to the bacterial movement is a non-monotonic function of the bacterial density (as in a liquid agar), but it also vanishes for extremely low densities. In this case it is clear that the specific functional form depend on the specific bacterial strain (B. subtilis., e.g., cannot move at all under such conditions).

\subsection{Chemotaxis}

Chemotaxis means changes in the movement of the cell in response to a gradient of certain chemical fields $[67-69,66]$. The movement is biased along the gradient either in the gradient direction or in the opposite direction. Bacteria are too short to estimate spatial gradients of the chemical by simply comparing concentrations at different 
locations on their membrane [68] (but see Ref. [70] for a different view). They deduce the spatial gradients by calculating temporal derivatives along their path. It is known that

E. coli, e.g., can compare successive measurements over a time interval of $3 \mathrm{~s}$. The actual chemotaxis in swimming bacteria is implemented by decreasing the tumbling frequency as cells swim up the gradient of the attractant or down the gradient of repellent. Thus the straight runs are important for gradient perception and the tumbling timing is important for the response to this gradient.

Usually chemotactic response means a response to an externally produced field like in the case of chemotaxis towards food. However, the chemotactic response can be also to a field produced directly or indirectly by the bacterial cells. We will refer to this case as chemotactic signaling.

Chemotaxis towards high concentration of nutrients is a well studied phenomenon in bacteria [67,71]. When the center of a soft agar plate $(0.35 \%$ agar concentration) is inoculated with cells capable of chemotaxis, distinct circular bands of bacterial cells become visible after a few hours of incubation. In fact, these patterns were used as semi-quantitative indicators of chemotactic response [71]. Genetic experiments showed that the creation of each of those bands depends solely on the chemotactic response to a single chemical in the substrate (these chemicals are usually metabolizable, but even cells that have lost the ability to metabolize a certain chemical form bands, as long as they are attracted to it [67]). Berg et al. [72] showed that the bacteria realize chemotactic response by modulating the periods between tumbling events they decrease the probability of tumbling when moving in a preferred direction along the chemical gradient. This makes a bias in the random walk which result in a mean drift of the bacteria in the desired direction, a drift that can be as large as $v / 10$.

Bacteria sense the local concentration $C$ of a chemical via membrane receptors binding the chemical's molecules $[67,69]$. The cell measures the concentration by calculating the relative number of occupied receptors $N_{o} /\left(N_{o}+N_{f}\right)$, where $N_{o}$ and $N_{f}$ are the number of occupied and free receptors respectively. For a given chemical $C, N_{o}$ is determined by two characteristic times: the mean time of a receptor occupation $\tau_{o}$, a constant determined by internal cellular processes - and the mean time lapse when the receptor is free $\left(\tau_{f}\right)$. Since $\tau_{f}$ is inversely proportional to the concentration of the chemical (with the proportion coefficient determined by the receptor affinity to the chemical), we get

$$
\frac{N_{o}}{N_{f}+N_{o}}=\frac{\tau_{o}}{\tau_{f}+\tau_{o}}=\frac{C}{K+C},
$$

where $K \equiv\left(C \tau_{f}\right) / \tau_{o}$ is constant. It is crucial to note that when estimating gradients of chemicals, the cells actually measure changes in the receptors' occupancy $N_{o} /\left(N_{o}+N_{f}\right)$ and not in the concentration itself. Using Eq. (3) we obtain

$$
\frac{\partial}{\partial x}\left(\frac{N_{o}}{N_{o}+N_{f}}\right)=\frac{K}{(K+C)^{2}} \frac{\partial C}{\partial x} .
$$


This means that the chemical gradient times a factor $K /(K+C)^{2}$ is measured. This dependence in known as the "receptor law" [73]. For very high concentration the chemotaxis response vanishes due to saturation of the receptors. The chemotactic response also vanishes at the opposite limit of small concentration, as the concentration reception is masked by external and internal noises. This effect is not included in the receptor law, which should be changed accordingly.

The receptor law is needed to explain the bands reported by Adler [67,71]. It can be shown that linear chemotactic response to a nutrient cannot produce such bands. A non-linear response like the "receptor law" must be included for the bands to form. Moreover, high concentration of the attractant represses both the strength of the chemotactic response [67] and the velocity of the expanding band [74]. These observations are accounted for by the "receptor law" for chemotactic response if one assumes that the average gradient sensed by the cells is proportional to the initial concentration of the chemical $[67,74]$.

The bacterial flux due to chemotaxis can be described by

$$
\boldsymbol{J}_{\text {chem }} \equiv \zeta(\sigma) \chi(C) \nabla C,
$$

where $\chi(C) \nabla C$ is the gradient sensed by the cell (with $\chi(C)$ having the units of 1 over chemical's concentration) and $\zeta(\sigma)$ is the bacterial response to the sensed gradient (having the same units as a diffusion coefficient). $\chi(C)$ is usually taken to be either constant or the "receptor law".

The function of the bacterial response $\zeta$ is positive for attractive chemotaxis (movement towards high concentrations) and negative for repulsive chemotaxis. If the movement is in liquid and at low bacterial densities, then $|\zeta(\sigma)| \propto \sigma D_{b}$. In a lubrication fluid which effect the bacterial movement, the chemotaxis is effected in the same way the diffusion is; $|\zeta(\sigma, l)| \propto \sigma D_{b}(\sigma, l)$.

In the case of high bacterial densities, collisions between bacteria can disrupt both the perception of chemical gradient and the bacterial response. As the collisions prevent the bacteria from moving on a straight line between tumbling events, the effective response to chemotaxis is reduced.

\subsection{Food consumption, reproduction and starvation}

The $\mathscr{T}$ bacteria, like most bacteria, reproduce by fission of the cell into two daughter cells which are practically identical to the mother cell. The crucial step in the cell division is the replication of the genetic material and its sharing between the daughter cells. Haste replication of DNA might lead to many errors - most organisms limit the rate of replication to about 1000 bases per second. Thus the reproduction must take at least minimal reproduction time $\tau_{R}$. This reproduction time $\tau_{R}$ is about $25 \mathrm{~min}$ in Bacilli.

For reproduction, as well as for movement and other metabolic processes, bacteria and all other organisms need influx of energy. Any organism which does not get its energy directly from sunlight (by photo-synthesis) needs an external supply of food. 


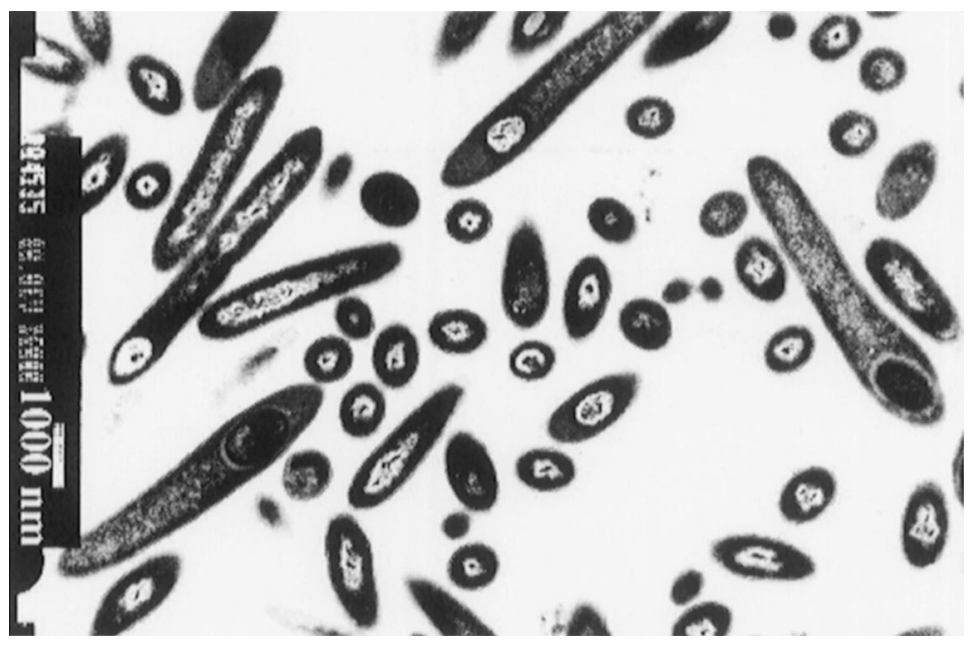

Fig. 8. Electron microscope observation of $\mathscr{T}$ bacteria. Round or oval shapes with bright center are spores. Elongated shapes are living cells. The cells engolfing oval shapes are pre-spores.

In the patterning experiments the bacteria eat nutrient from the agar. As long as there is enough nutrient and no significant amount of toxic materials, food is consumed (for cell replication and internal processes) at maximal rate $\Omega_{c}$. To estimate $\Omega_{c}$ we assume that a bacterium needs to consume an amount of food $C_{R}$ of about $3 \times 10^{-12} \mathrm{~g}$. It is three times its weight - one quanta for doubling body mass, one quanta used for movement and all other metabolic processes during the reproduction time $\tau_{R}$, and one quanta is for the reduced entropy of making organized cell out of food. Hence $\Omega_{c}$ is about $2 \mathrm{fg} / \mathrm{s}\left(1 \mathrm{fg}=10^{-15} \mathrm{~g}\right)$.

If nutrient is deficient for a long enough period of time, the $\mathscr{T}$ cells may enter a special stationary state - a state of a spore - which enables them to survive much longer without food. The bacterial cells employ very complex mechanisms tailored for the process of sporulation. They stop normal activity - like movement - and use all their internal reserves to metamorphose from an active volatile cell to a sedentary durable "seed". While the spores themselves do not emit any chemicals (as they have no metabolism), the pre-spores (sporulating cells, see Fig. 8) do not move and emit a very wide range of waste materials, some of which unique to the sporulating cell. These emitted chemicals might be used by other cells as a signal carrying information about the conditions at the location of the pre-spores. Ben-Jacob et al. $[27,62,75]$ suggested that such materials are repelling the bacteria ("repulsive chemotactic signaling") as if they escape a dangerous location.

When bacteria are grown in a petri dish, nutrients are usually provided by adding peptone, a mixture including all the amino acids and sugars as source of carbon. Bacteria which are not defective in synthesis of any amino acid can grow also on a minimal agar in which a single source of carbon and no amino acids are provided. Such growth might seem to be easier to model as the growth is limited by the diffusion 
of a single chemical. However, during growth on minimal agar there is usually a higher rate of waste products accumulation, introducing other complications into the model. Moreover many of our strains are auxotrophic i.e. defective in synthesis of some amino acids and need an external supply of it. Providing the bacteria with these amino acids and only a single carbon source might pose us the question as to what is the limiting factor in the growth of the bacteria. For all those reasons we prefer to use peptone as nutrient source.

We said that if there is ample supply of food, bacteria reproduce in a maximal rate of one division in $\tau_{R}$. If the available amount of food is limited, bacteria consume the maximum amount of food they can. In the limit of low bacterial density, the available amount of food over the tumbling time $\tau_{T}$ is the food contained in the area $\tau_{T} \sqrt{D_{b} D_{n}}$, where $D_{b}$ and $D_{n}$ are the diffusion coefficients of the bacteria and the food, respectively. Hence the rate of food consumption is given by $n \sqrt{D_{b} D_{n}}$ (whether $D_{b}$ is constant or not).

In a continuous model, reproduction of bacteria translate to a growth term of the bacterial density which is $\sigma$ times the eating rate per bacteria. In the limit of high nutrient it is $\sigma / \tau_{R}$, and in the limit of low nutrient it is proportional to $n \sigma$. This brings to mind Michaelis-Menten law [73] of $[K /(1+\gamma n)] n \sigma$ with $K, \gamma$ constants. Many authors take only the low nutrient limit of this expression, $K n \sigma$, although it is not biologically established that the bacteria in the experiments are limited by the availability of food and not by their maximal consumption rate.

\section{Reaction-diffusion models}

In this section we deal with continuous, reaction-diffusion models for bacterial growth. The models under study are due to Fisher and Kolmogorov [76,77], Kessler and Levine [61], Kitsunezaki [59], Kawasaki et al. [58] and Mimura et al. [56,57]. The models are all two-dimensional (2D), with $b(\mathbf{x}, t)$ denoting the density of bacteria projected on a $2 \mathrm{D}$ plane and $n(\mathbf{x}, t)$ is the $2 \mathrm{D}$ nutrient density. The equations for the various models will be written in dimensionless units, and the reader is referred to the Appendix for a discussion about the relations with real units.

In general, the rate of change of the bacteria density can be described by [73]

$$
\frac{\partial b}{\partial t}=\text { movement }+ \text { "birth" - "death" }
$$

As discussed in Section 3, the movement of bacteria consists of various possible mechanisms, of which we will concentrate on swimming, so that the motion is described as diffusion (either linear or non-linear). The "birth" term in Eq. (6) corresponds to bacterial reproduction, which depends on the supply of nutrients. The "death" term represents the transition of bacteria into a non moving state. 

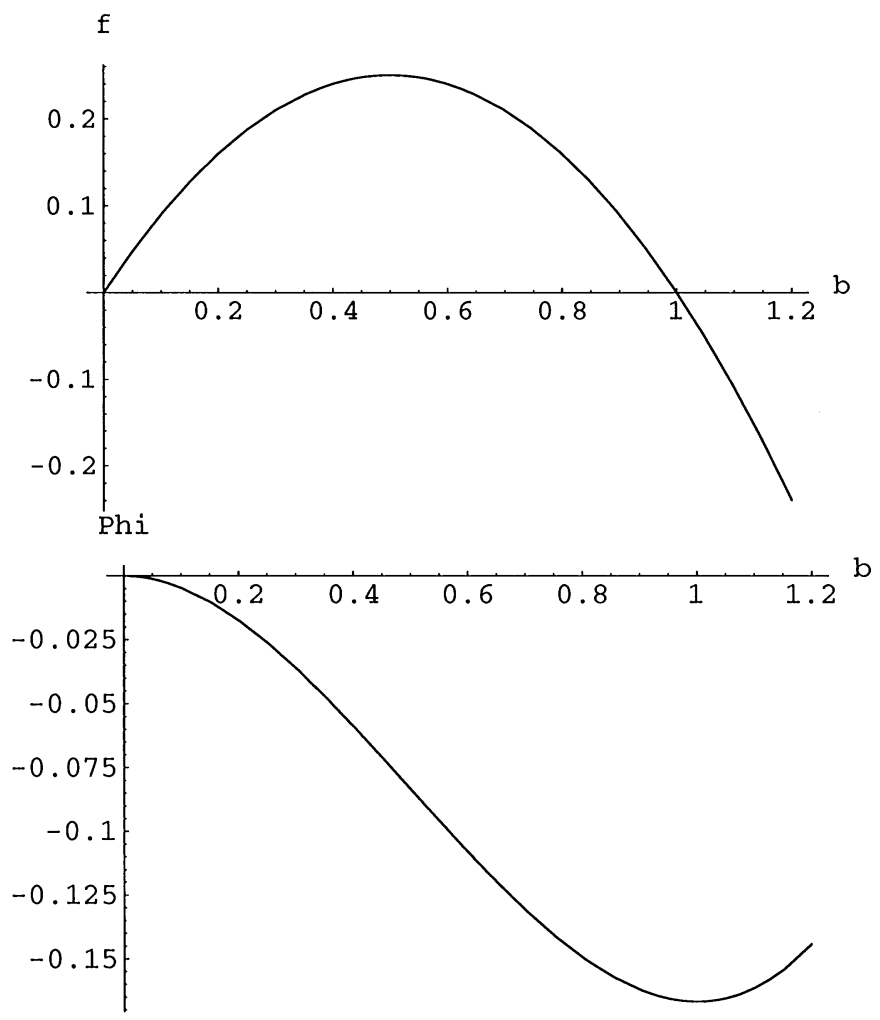

Fig. 9. Reaction term $f(b)$ (above) and Landau-Ginzburg free energy $\Phi(b)$ (below) for the Fisher-Kolmogorov equation.

\subsection{The Fisher-Kolmogorov equation}

Mathematically, the above description is usually written as a reaction diffusion equation, for which the canonic example is the Fisher-Kolmogorov equation [76,77] (without a death term):

$$
\frac{\partial b}{\partial t}=D_{b} \nabla^{2} b+b(1-b) \text {. }
$$

This equation was originally presented to describe the spread of mutants in a population. We will use it here as a starting point for our discussion of colonial development. In this context, $D_{b}$ is the diffusion coefficient describing the bacterial movement, and the reaction term $f(b)=b(1-b)$ describes both the growth and "death" of bacteria. The function $f(b)$ is depicted in Fig. 9. Eq. (7) has two homogeneous solutions, a stable solution $b=1$ and an unstable solution $b=0$. These solutions correspond to the two extrema of the potential $\Phi=-\int f(b) d b$, which can be thought of as a LandauGinzburg free energy density (see Fig. 9). Thus, we can study the propagation of the stable state (inside the colony) into the unstable one (outside the colony). It can be 


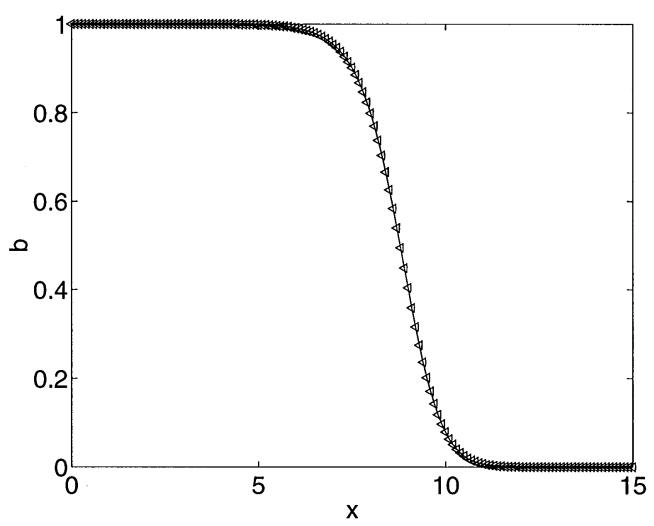

Fig. 10. Typical front obtained for the 1D diffusive Fisher-Kolmogorov model. Parameters: $D_{b}=0.1, D_{n}=1$, Initial food concentration $n_{0}=1$.

shown [78-83] that in 1D there is a unique selected velocity of the front, chosen according to the properties of the fixed point far ahead at infinity.

In two dimensions, the propagation is in the form of a compact (as opposed to branching) growth. That is, there is no Mullins-Sekerka instability [41,84-86]. In the case of such instability, a small bump in a flat interface will have a higher velocity than the rest of the front and will therefore over-grow. Here, however, this will not happen, because the front velocity is determined by the fixed point at infinity, rather than by local properties of the front.

The Fisher-Kolmogorov model is an appropriate description of the growth when bacteria are grown under nutrient-rich conditions, in which case the growth dynamics is not limited by the supply of food [87]. Here we are interested in the opposite case, where nutrient supply is limited. For a more realistic description of colonial development on a nutrient-poor surface, we must take into account the interaction of bacteria with the nutrient field $n(\mathbf{x}, t)$. In the simplest case, this is described by the Diffusive Fisher-Kolmogorov equation [85,86]:

$$
\begin{aligned}
& \frac{\partial b}{\partial t}=D_{b} \nabla^{2} b+f(b, n), \\
& \frac{\partial n}{\partial t}=D_{n} \nabla^{2} n-\eta f(b, n),
\end{aligned}
$$

where $\eta>0$ is the conversion ratio of food into bacteria (3 picogram per bacteria, see Section 3). In this model the food consumed by the bacteria is reduced from the food field and converted into bacteria. The shape of a one-dimensional front obtained for $f(b, n)=b n$ is depicted in Fig. 10. It turns out that this model (as the original Fisher-Kolmogorov model) has a selected front velocity, determined by the conditions at infinity, and therefore does not exhibit diffusive (Mullins-Sekerka) instability, and a two dimensional growth is compact rather than branched. An additional way of understanding why compact growth is obtained even under poor nutrient levels is to 


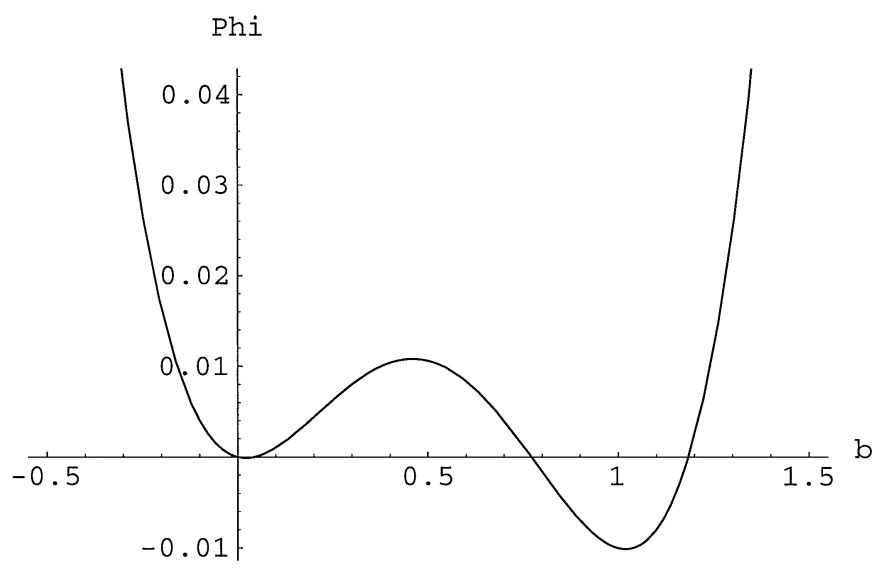

Fig. 11. Landau-Ginzburg free energy $\Phi(b)$ for the solid-liquid phase-field model. Note the meta-stable point at $b=0$ and the stable one at $b=1$.

note that the model, as it is, does not impose a minimal bacteria density in the colony, so that the density can be adjusted according to the initial food level. In the real biological system, however, some minimal density is required in order to create the lubricating fluid (needed for movement), and so compact growth usually is not possible.

For further understanding the requirements for a branching pattern, let us recall the case of solidification from an under-cooled melt, which exhibits branching patterns $[47,48]$. Our bacterial density $b$ corresponds to the order parameter in the phase-field model description of solidification $[88,89]$, whereas the diffusion of food is analogous to the diffusion of heat away from the solid-liquid interface. In the case of solidification, the Landau-Ginzburg free energy is a tilted double-well (see Fig. 11). The metastable state corresponds to the liquid phase. In the diffusive Fisher-Kolmogorov case, the analog of the liquid state, i.e. the $b=0$ state, is unstable. Thus, according to the solidification case, if we modify the model and turn the $b=0$ state into a meta-stable one, this can lead to branching growth.

\subsection{A cutoff in the reaction term}

In the case of bacteria, there is a feature of the system that might have a similar effect to the meta-stability in solidification. This is the discreteness of bacteria, for which the continuous description is not always valid. Kessler and Levine [61] argue that when describing a discrete system using continuous models, a cutoff near the fixed point must be imposed, i.e. the reaction term must be set to zero when the (bacterial-) density is below some threshold. They have shown that inclusion of such a cutoff leads to a Mullins-Sekerka instability, and branching patterns may appear when a death term is also included, as explained below. 


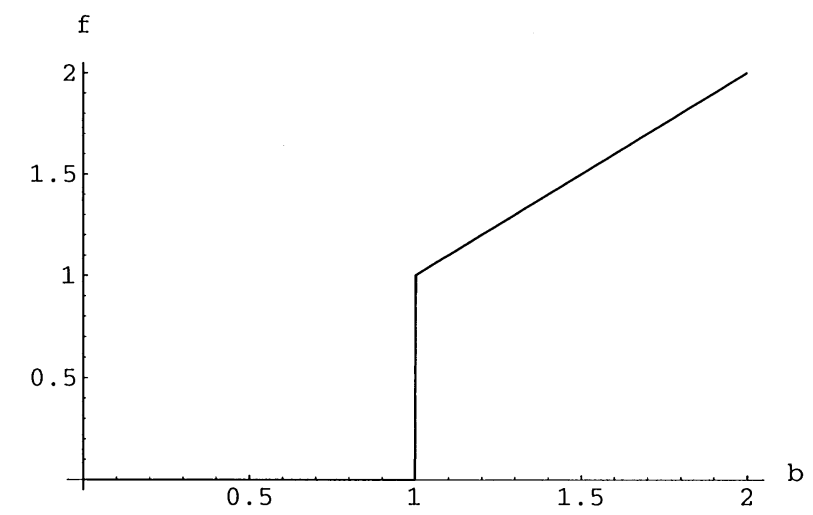

Phi

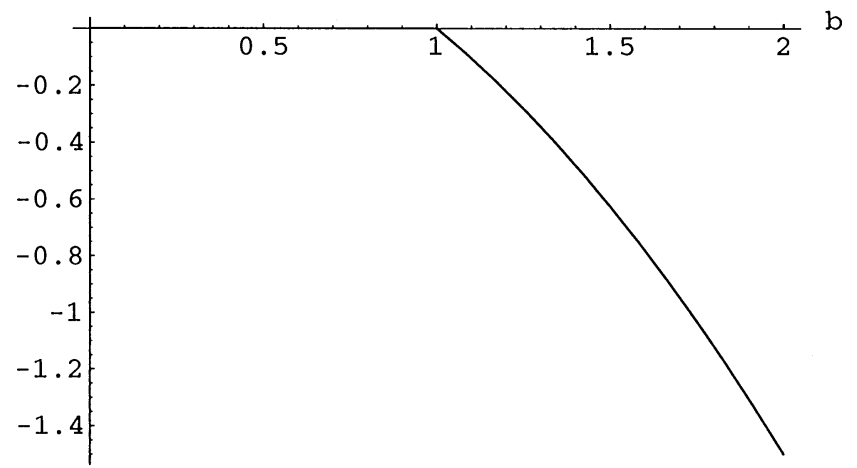

Fig. 12. Growth term $f(b)$ (above) and Landau-Ginzburg free energy $\Phi(b)$ (below) for the Kessler-Levine correction. Cutoff value $\beta=1$, nutrient level $n=1$.

To show this, we consider the diffusive Fisher equations with a cutoff:

$$
\begin{aligned}
& \frac{\partial b}{\partial t}=D_{b} \nabla^{2} b+b n \Theta(b-\beta), \\
& \frac{\partial n}{\partial t}=\nabla^{2} n-n b \Theta(b-\beta),
\end{aligned}
$$

where $\beta$ is the threshold density for growth, and $\Theta$ is the Heaviside step function. The food consumption term is of the form $f(n, b)=n b$, which is the widely used lownutrient approximation for the Michaelis-Menten law (Section 3.4). The value of the threshold for bacterial growth, $\beta$, is taken to be one bacterium per $1-10 \mu \mathrm{m}^{2}$. Note that this corresponds to the case where the distance between bacteria is of the order of the length between tumbles. Fig. 12 depicts the reaction term and the Landau-Ginzburg free energy for this model. As can be seen in Fig. 13, an instability of the front indeed appears, and the compact growth pattern has a surface broken by "fjords". However, the Mullins-Sekerka instability is not sufficient to produce branches. The emerging dips soon "heal", so that branches are not formed. One way to obtain branching growth is 

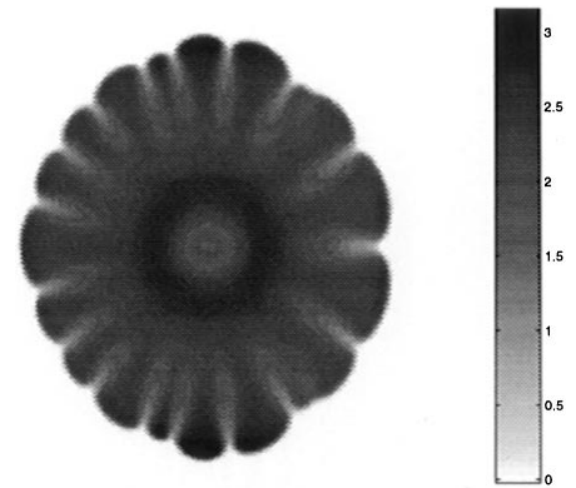

Fig. 13. 2D growth pattern of the Kessler-Levine correction with no death term. Parameters are: $D b=0.01, \beta=0.25, n_{0}=1$.
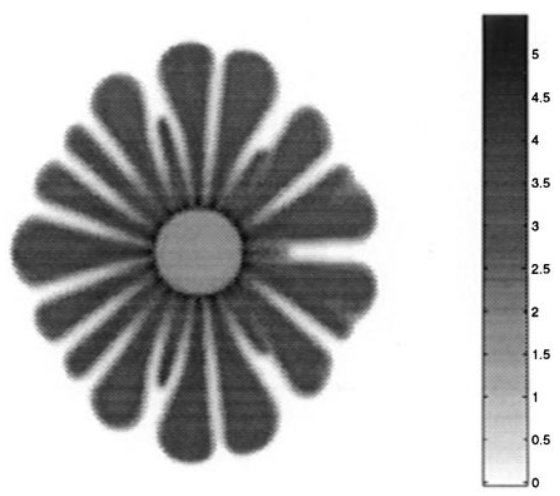

Fig. 14. 2D growth pattern $(b+s)$ of the Kessler-Levine correction with a death term. Parameters are: $D b=0.01, \beta=0.25, n_{0}=1, \mu=0.01$.

to add a "death" term to the model, thus

$$
\begin{aligned}
& \frac{\partial b}{\partial t}=D_{b} \nabla^{2} b+b n \Theta(b-\beta)-\mu b, \\
& \frac{\partial n}{\partial t}=\nabla^{2} n-n b \Theta(b-\beta), \\
& \frac{\partial s}{\partial t}=\mu b,
\end{aligned}
$$

where $\mu$ is the rate of bacterial differentiation into non-moving state, and $s(\mathbf{x}, t)$ is the density of "frozen" bacteria. This modified model exhibits distinct branching patterns, as seen on Fig. 14. The explanation for this effect lies in the fact that now, with a death term present, bacteria left behind the propagating front become non-motile ("dead"). They are unable to move and close the "fjords", thus allowing real branches to form. 


\subsection{Reaction-diffusion with lubrication}

We have so far ignored the effect of the lubricating field on the motion of the bacteria. We present here a new model which incorporates an additional field that describes the lubricating fluid. The field, denoted as $l$, is the local height of the lubrication fluid on the agar surface. Its dynamics is governed by a reaction diffusion equation. The bacterial diffusion is coupled to this field.

The dynamics of the field is given by

$$
\frac{\partial l}{\partial t}=-\nabla \boldsymbol{J}_{l}+\Gamma b n\left(l_{\max }-l\right)-\lambda l,
$$

where $\boldsymbol{J}_{l}$ is the fluid flux (to be discussed), $\Gamma$ is the production rate and $\lambda$ is the absorption rate of the fluid by the agar.

We assume that the fluid production depends on the bacterial density. As the production of lubrication probably demands substantial energy, it should also depend on the nutrients level. We assume that the absorption of fluid into the agar depends on the local amount of fluid (i.e. the height of the fluid layer). In this model the production depends linearly on the concentrations of both the bacteria and the nutrients. The production term cannot become negative as the lubrication height cannot exceed $l_{\max }$.

The lubrication fluid flows by diffusion and by convection caused by bacterial motion. A simple description of the convection is that each bacterium drags along its movement the fluid surrounding it:

$$
\boldsymbol{J}_{l}=-D_{l} l^{\eta} \nabla l+j \boldsymbol{J}_{b},
$$

where $D_{l}$ is the lubrication diffusion coefficient, $\boldsymbol{J}_{b}$ is the bacterial flux and $j$ is the amount of fluid dragged by each bacterium. The diffusion term of the fluid depends on the height of the fluid to the power $\eta$. The nonlinearity causes the fluid to have a sharp boundary at its front, as is observed in the experiments of bacterial colonies development.

We now turn to the effect of the lubrication on bacterial diffusion. An increase in the amount of lubrication decreases the friction between the bacteria and the agar surface. The term "friction" is used here in a very loose manner to represent the total effect of any force or process that slows down the bacteria. It might include, for example, the drag which acts on a body moving in shallow layer of viscous fluid. It might include the probability that a flagellum will adhere or get tangled with the polymers of the agar. As the bacterial motion is over-damped, the local speed of the bacteria (or the mean step length, when neglecting collisions between bacteria) is proportional to the self-generated propulsion force divided by the friction. It can be shown that variation of the step length leads to variation of the diffusion coefficient, with the diffusion coefficient proportional to the step length to the power of two. We assume that the friction is inversely related to the local lubrication height through some power law: friction $\sim l^{\gamma}$ and $\gamma<0$. Following our arguments the bacterial flux is

$$
\boldsymbol{J}_{b}=-D_{b} l^{-2 \gamma} \nabla b \text {. }
$$


(a)

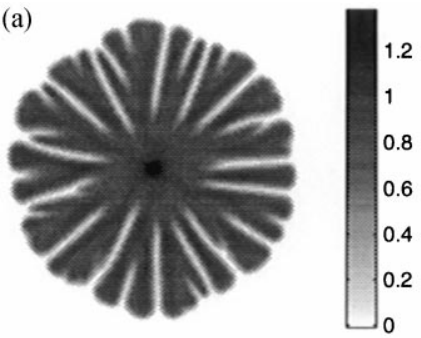

(c)

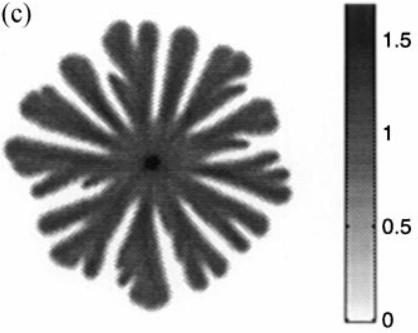

(b)

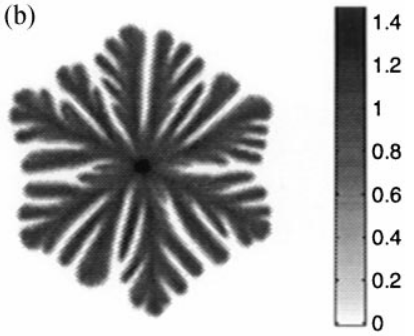

(d)

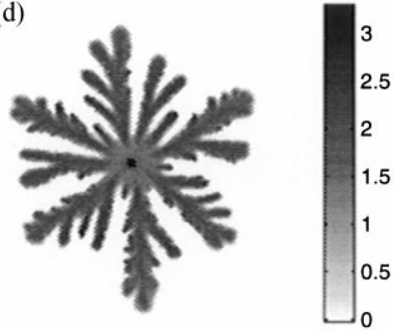

Fig. 15. 2D growth patterns $(b+s)$ of the model with lubrication. (a) Parameters are $\gamma=-1 / 2, \lambda=0.01, \eta=2, D_{b}=0.5, D_{l}=0.5, \mu=1, n_{0}=1.5, j=0.01, l_{\max }=2, \Gamma=1$. (b) Parameters are as in (a), except a higher absorption rate $\lambda=0.1$. (c) Parameters are as in (a), except a different exponent for the bacterial diffusion $\gamma=-1$. (d) Parameters are as in (c), except a higher absorption rate $\lambda=0.03$.

For the complete model we took simple bacterial growth and death terms. The model is

$$
\begin{aligned}
& \frac{\partial b}{\partial t}=D_{b} \nabla\left(l^{-2 \gamma} \nabla b\right)+b n-\mu b, \\
& \frac{\partial n}{\partial t}=\nabla^{2} n-b n, \\
& \frac{\partial l}{\partial t}=\nabla\left(D_{l} l^{\eta} \nabla l+j D_{b} l^{-2 \gamma} \nabla b\right)+\Gamma b n\left(l_{\max }-l\right)-\lambda l, \\
& \frac{\partial s}{\partial t}=\mu b .
\end{aligned}
$$

For the initial condition, we set

$$
n(\mathbf{x}, t)=n_{0}, \quad b(\mathbf{x}, t)=b_{0}(\mathbf{x}),
$$

where $n_{0}$ is the initial concentration of nutrients and $b_{0}(\mathbf{x})$ is the initial bacterial distribution. In the simulations, $b_{0}(\mathbf{x})$ is localized at the center.

Preliminary results show that the model can reproduce branching patterns (Fig. 15). At low values of the absorption rate the model exhibits dense fingers. At higher values of the absorption rate the model exhibits finer branches. We obtain finer branches also if we change other parameters that effectively decrease the amount of lubrication. We 

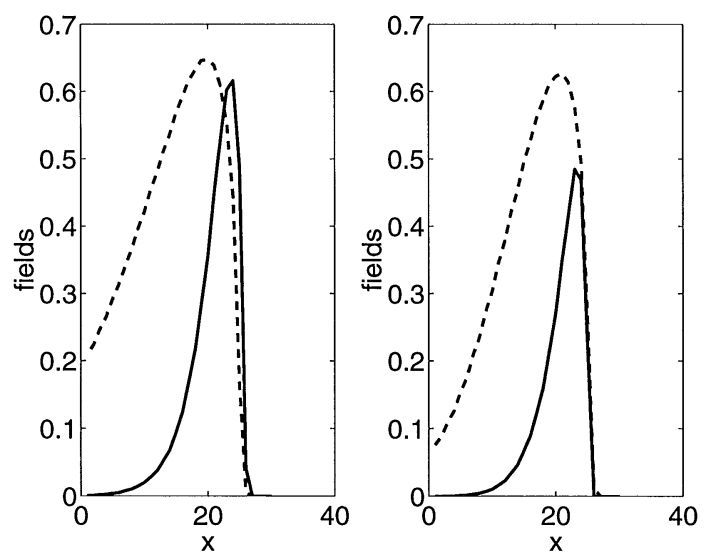

Fig. 16. Profile of the fronts of the bacterial field (solid line) and of the lubricating field (dashed line) from the 2D model with lubrication. The bacterial field was scaled by a factor of 40 . Left: Parameters are as in Fig. 15a. Right: Parameters are as in Fig. 15c.

can relate these conditions to high agar concentration. In this model, as in the nonlinear diffusion model described below, the bacterial field and the lubricating field have a front "wall" with compact support (Fig. 16).

\subsection{Non-linear diffusion}

It is possible to introduce a simplified model, where the fluid field is not included, and is replaced by a density-dependent diffusion coefficient for the bacteria $D_{b} \sim b^{k}$ $[90,91]$. For this purpose, a few assumptions are needed about the dynamics at low bacterial and lubrication density:

- The production of lubricant is proportional to the bacterial density to the power $\alpha>0$.

- There is a sink in the equation for the time evolution of the lubrication field, e.g. absorption of the lubricant into the agar. This sink is proportional to the lubrication density to the power $\beta>0$.

- Over the bacterial length scale, the two processes above are much faster than the diffusion process, so the lubrication density is proportional to the bacterial density to the power of $\beta / \alpha$.

- The friction is proportional to the lubrication density to the power $\gamma<0$.

Given the above assumptions, the lubrication field can be removed from the dynamics and be replaced by a density dependent diffusion coefficient. This coefficient is proportional to the bacterial density to the power $k \equiv-2 \gamma \beta / \alpha>0$.

A model of this type is offered by Kitsunezaki [59]:

$$
\frac{\partial b}{\partial t}=\nabla\left(D_{0} b^{k} \nabla b\right)+n b-\mu b,
$$




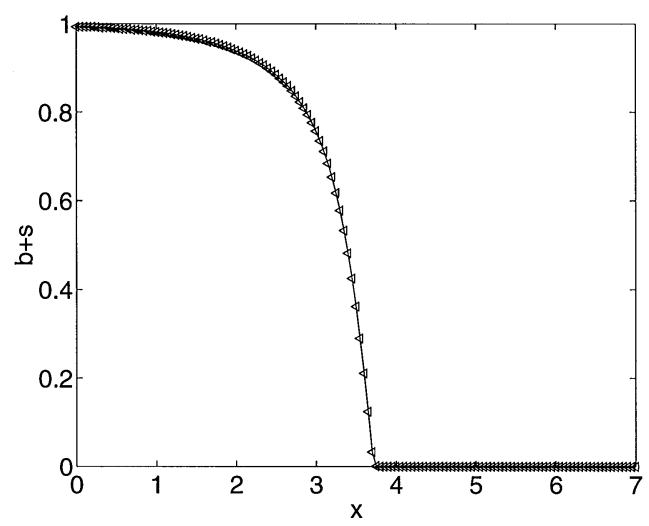

Fig. 17. 1D front obtained for the Kitsunezaki model. Parameters are: $D_{0}=0.1, k=1, \mu=0.15, n_{0}=1$.

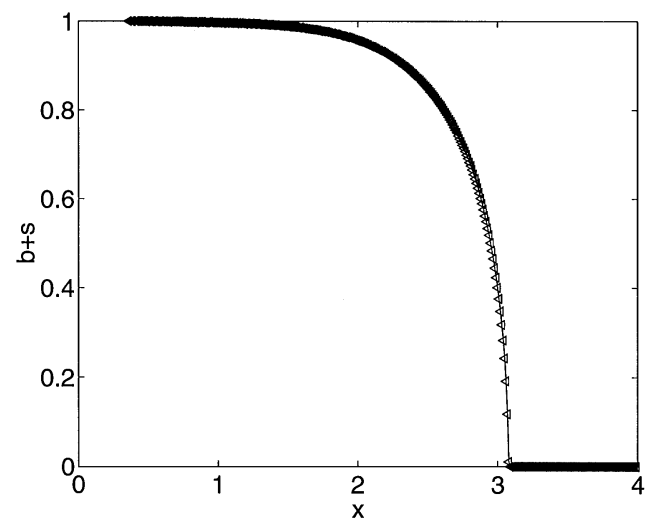

Fig. 18. 1D front obtained for the Kitsunezaki model with $k=2$. All other parameters as in Fig. 17 .

$$
\begin{aligned}
& \frac{\partial n}{\partial t}=\nabla^{2} n-b n, \\
& \frac{\partial s}{\partial t}=\mu b .
\end{aligned}
$$

For $k>0$ the 1D model gives rise to a front "wall", with compact support (i.e. $b=0$ outside a finite domain, see Fig. 17). For $k>1$ this wall has an infinite slope (Fig. 18). The propagation velocity in this case is determined by the condition at the front, not at infinity [90,92]. We therefore expect a Mullins-Sekerka instability in 2D (as is claimed in [59]). Indeed, the model exhibits branching patterns for suitable parameter values and initial conditions (Fig. 19). Note, that the compact support exhibited by this model (that is, the abrupt disappearance of bacterial presence outside the colony boundary) is much more in accordance with experimental observations than the long "tail" of bacterial density appearing in the Fisher-Kolmogorov case (recall Fig. 10). 

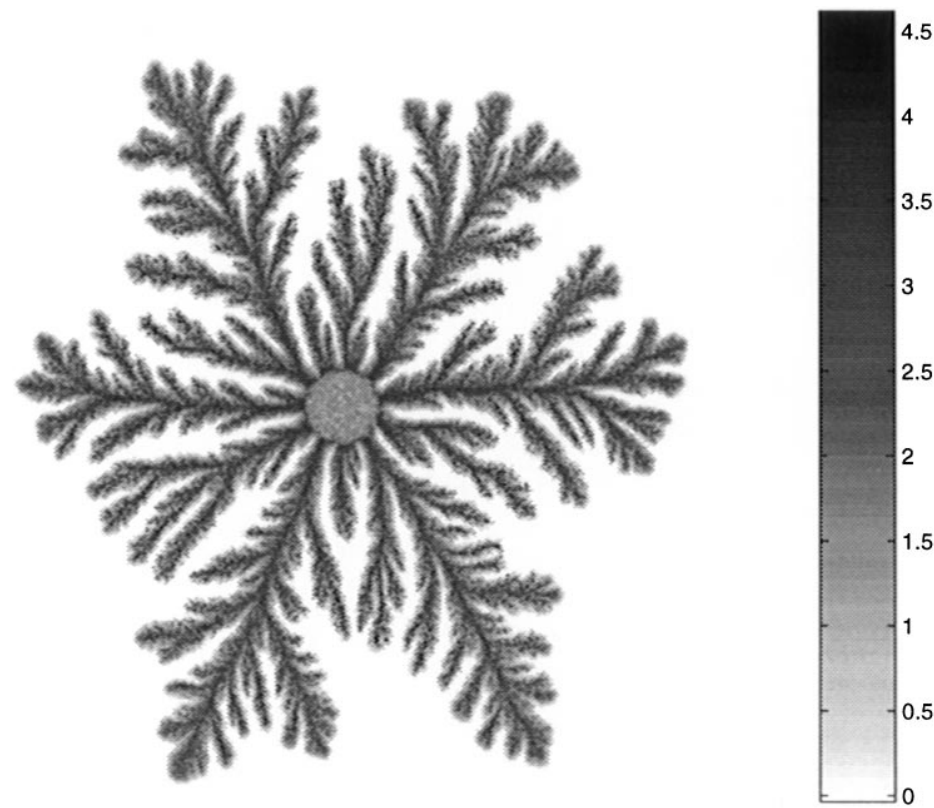

Fig. 19. 2D growth pattern $(b+s)$ of the Kitsunezaki model. Parameters are: $D_{0}=0.1, k=1, \mu=0.15$, $n_{0}=1$.

Another state-dependent diffusion coefficient was proposed and studied by Kawasaki et al. [58], which took $D_{b} \sim n b$. They justify this form by the observation that bacteria are active mostly at the edge of the colony - the only area where there is high amount of bacteria and food. Their model, too, exhibits branching shapes (see Fig. 20). This is due to the $b$ dependence of the diffusion coefficient, which leads to front instability, just as in the Kitsunezaki model. The fact that $D_{b}$ also depends on $n$ prevents bacteria inside the colony from moving - and closing the dips created by the instability. In this way, branches are created without a need for a death term. A similar mechanism of "food" dependent diffusion coefficient was used by Tu et al. [93], who describe a mean-field model for DLA. Their model, too, does not include a death term yet produces branching patterns.

\subsection{A meta-stable reaction term}

As mentioned earlier, meta-stability of the growth term can lead to branching patterns. Mimura et al. [56,57] have studied the following model, for which $b=0$ is a meta-stable fixed point:

$$
\frac{\partial b}{\partial t}=D_{b} \nabla^{2} b+\varepsilon b n-\frac{\mu b}{(b+1)(n+1)},
$$



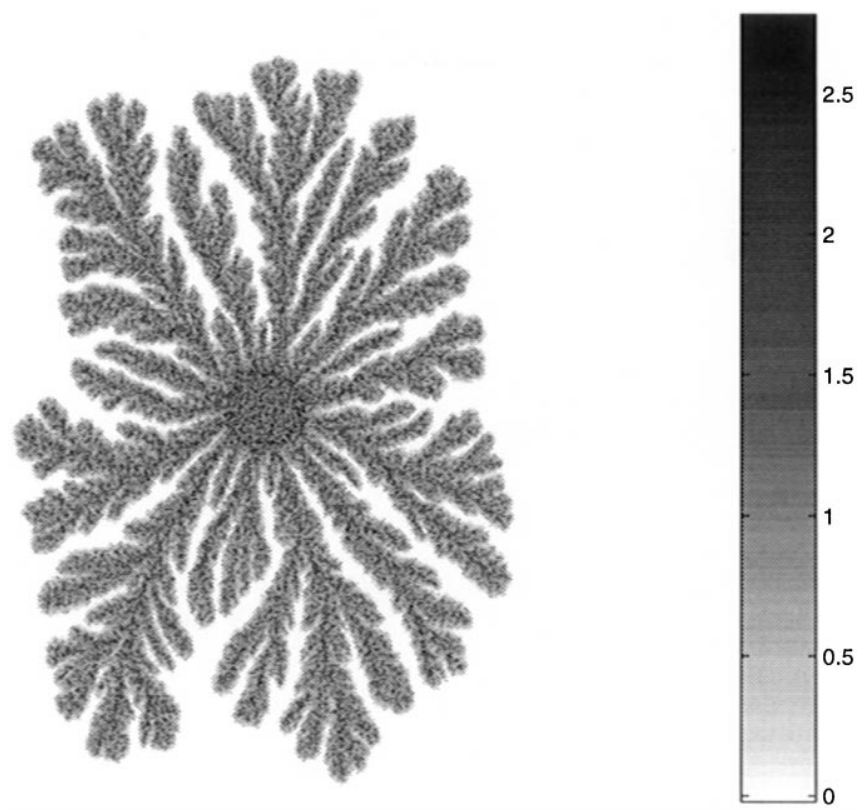

Fig. 20. 2D growth pattern (b) of the Kawasaki et al. model. Parameters are: $D_{b}=n * b, n_{0}=0.71$.

$$
\begin{aligned}
& \frac{\partial n}{\partial t}=\nabla^{2} n-n b, \\
& \frac{\partial s}{\partial t}=\frac{\mu b}{(b+1)(n+1)}
\end{aligned}
$$

(see the Appendix for relations to real units). In this model, $D_{b}$ does not depend on bacterial density, and its value is said to vary with agar concentration. The key feature of this model is that the total bacteria growth term (multiplication minus inactivation), depicted in Fig. 21, gives a meta-stable state at $b=0$. This means that in order to initiate bacterial growth, a threshold value of $b^{*}=\{\mu /[\varepsilon n(n+1)]\}-1$ must be reached (this value corresponds to bacterial density of the order of 0.1 bacteria $/ \mu \mathrm{m}^{2}$. This feature contradicts the observations, that even a small number of bacteria, inoculated on a substrate, will multiply and later begin to move. This does not imply that the model is incorrect. A possible interpretation is that the field $b$ actually describes a combination of lubricant + bacteria. In this case, however, as we have explained before, we would expect the model to exhibit a non-linear diffusion. Hence we believe that this model might provide a better description of the bacteria if the diffusion term is replaced with a non-linear diffusion term. Fig. 22 depicts a 1D front obtained for the model. The model as it is exhibits various branching patterns, patterns of concentric rings and compact growth (Fig. 23).

Mimura et al. argue that the model captures the experimental morphology diagram they observed. This is a very crucial point. If indeed the above claim is correct, it implies that the observed patterns can be reproduced with no need for additional 

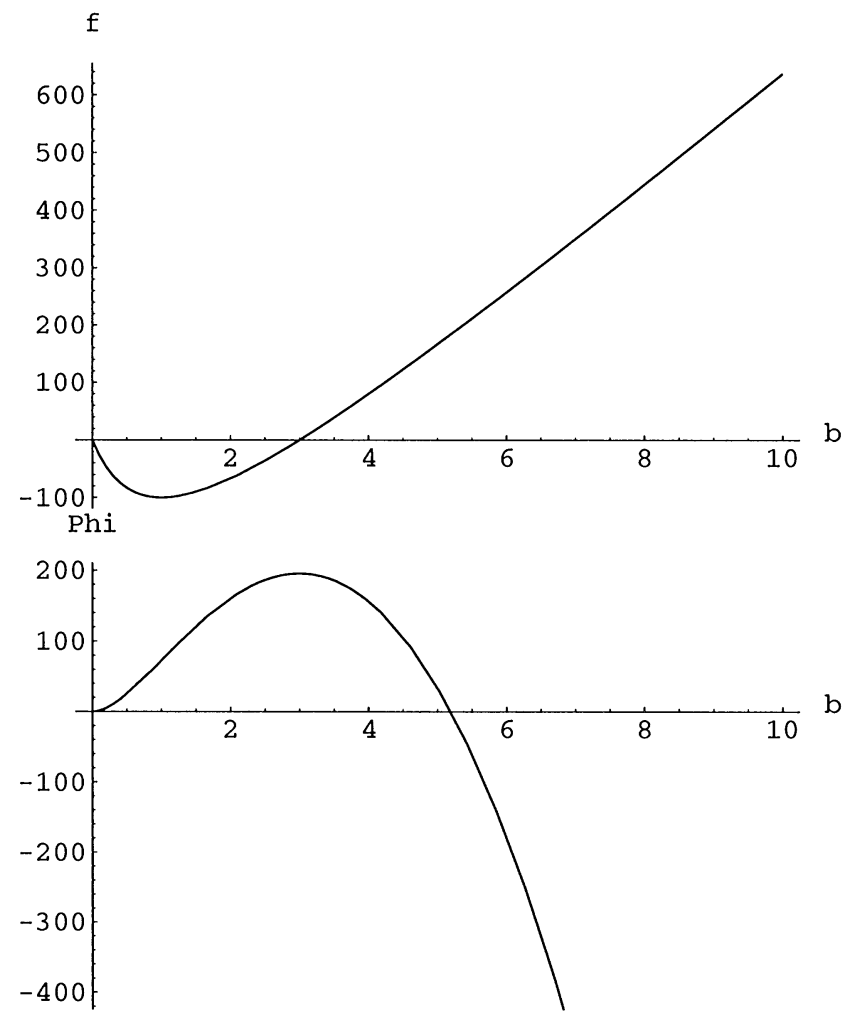

Fig. 21. Growth term $f(b)$ (above) and Landau-Ginzburg free energy $\Phi(b)$ (below) for the Mimura et al. model. Parameters are: $\varepsilon=20, \mu=2400, n=5$. Note the stable point at $b=0$.

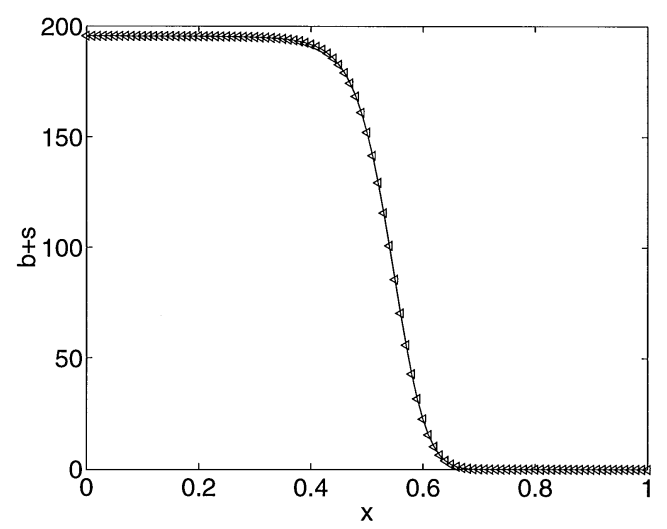

Fig. 22. 1D front obtained for the Mimura et al. model. Parameters are: $D_{b}=0.1, n_{0}=10, \varepsilon=20, \mu=2400$.

biological features. However, using the discrete communicating walkers model [27], Ben-Jacob et al. have concluded that the additional features of chemotactic response have to be included. So, in order to check this point we have performed more detailed comparison between the Mimura et al. model and the experimental observations. 
(a)

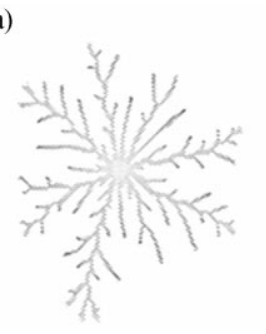

(c)

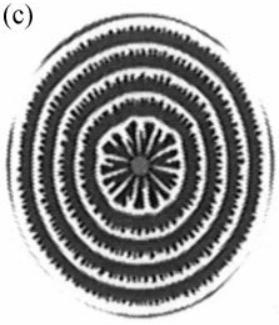

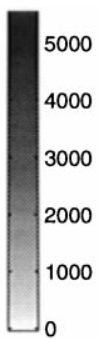

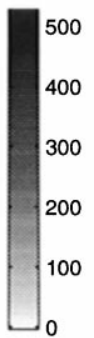

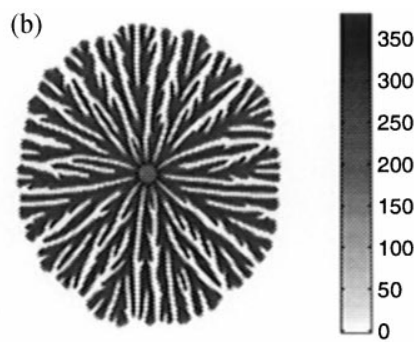

(d)

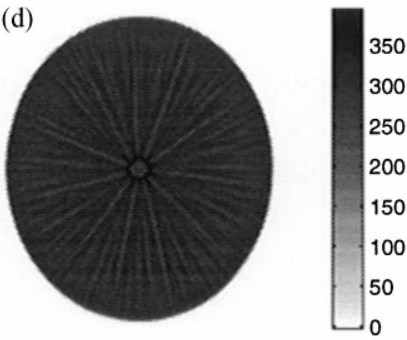

Fig. 23. Various 2D patterns $(b+s)$ obtained for the Mimura et al. model: (a) DLA-like $\left(D_{b}=0.05, n_{0}=10\right)$. (b) dense branches $\left(D_{b}=0.09, n_{0}=10\right)$. (b) Concentric rings $\left(D_{b}=0.05, n_{0}=12\right)$. (c) Compact growth $\left(D_{b}=0.1, n_{0}=14\right)$. In all cases, $\mu=2400, \varepsilon=20$.

First, we consider the DLA-like growth. In this case, the bacteria do not move on the agar surface, and the growth is indeed very similar to the DLA algorithm, as was proposed by Matsushita et al. [33,37]. It is now understood that in a mean-field DLA model the particle density (density of bacteria in the present case) can not be described by a diffusion term. Instead, it has to be described by a diffusion multiplied by the nutrients field [93], which differs from the linear diffusion in the model of Mimura et al. Indeed, close inspection of the fractal pattern created by the model reveals that it differs from the observed DLA-like patterns.

Another test of the model is the predicted pattern of concentric rings. It has already been pointed out by Rafols [60] that the model's pattern differs from the observed one. In the experiment, branching growth slows down. The branches become wider and growth stops. Then, after bacterial differentiation, a new cycle of branches growth starts with thin branches emitted from the stationary wide branches [60]. This description differs from the model patterning shown in Fig. 23.

In Fig. 24 we exhibit results of numerical simulations for various levels of peptone and for agar concentration for which concentric rings are observed. The sequence of patterns from DLA-like at low peptone to concentric rings at high levels of peptone differ from the similar sequence of observed patterns presented in Ref. [60].

We have also tested the change in patterns as we vary the agar concentration (see Fig. 25). When we plot the growth velocity as a function of the agar concentration 

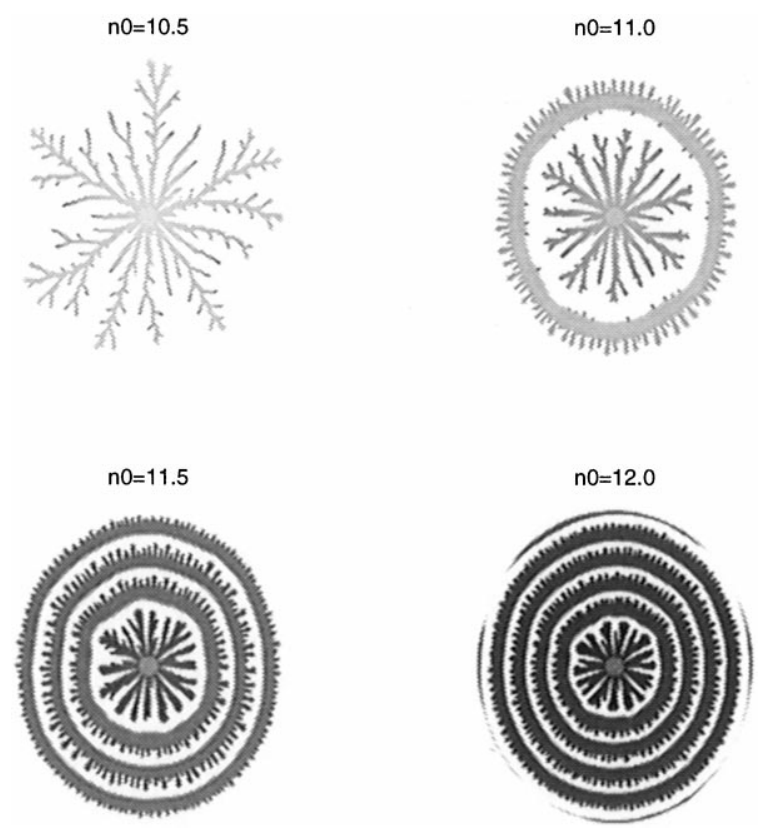

Fig. 24. Mimura et al. model: The effect of changing the initial nutrient level $n_{0}$. For all pictures $D_{b}=0.05, \mu=2400, \varepsilon=20$.

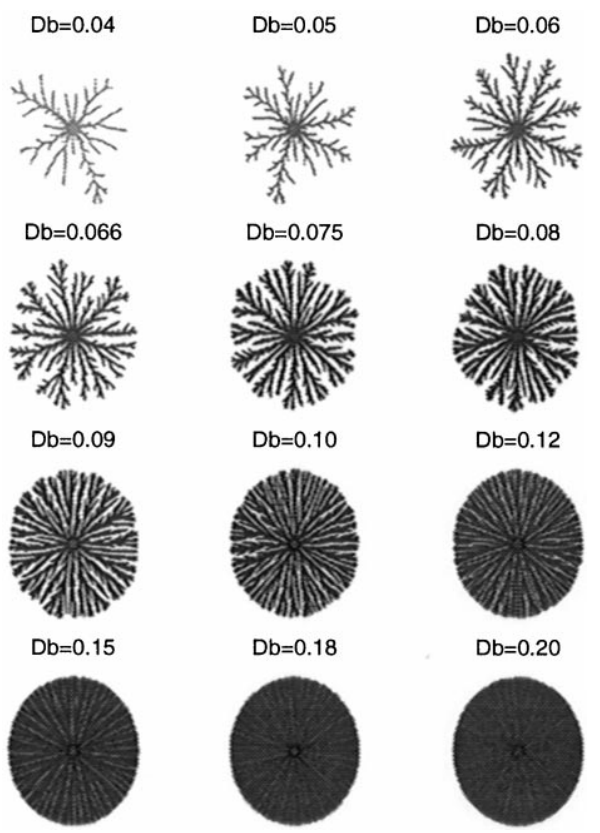

Fig. 25. Mimura et al. model: The effect of changing $D_{b}$. For all pictures $n_{0}=10, \mu=2400, \varepsilon=20$. 


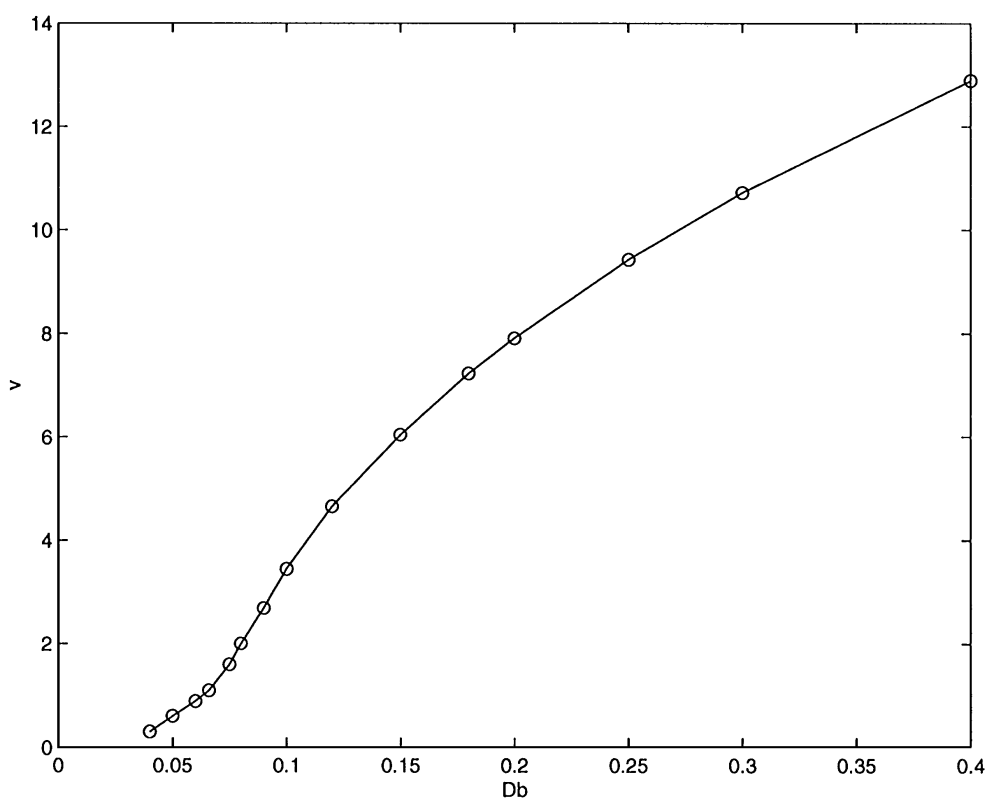

Fig. 26. Colonial growth velocity vs. $D_{b}$ for the Mimura et al. model. Parameter values as in Fig. 25.

(Fig. 26), it does not show a jump in the velocity or its slope. In other words, the model seems to exhibit a simple crossover between the patterns rather than a morphology transition as the observations seem to indicate.

The above results lead us to conclude that the Mimura et al. model does capture some of the observed branching patterns, yet the complete description of the observations requires additional features to be included. Specifically, we propose to include nonlinear diffusion. We also believe that chemotactic response does play an important role for poor growth conditions. To further test this conclusion, we present in the next section a study of patterns produced by the reaction-diffusion models when chemotactic responses are included.

\section{Incorporation of chemotactic signaling in the continuous models}

So far we have seen several models for the branching colonies, each with its own mechanism of diffusive instability, which produces patterns resembling the observed ones. Is there a way to distinguish between the models so as to find out what are the biological features underlying the diffusive instability? We rely on results from an atomistic model - the Communicating Walkers model [27,94,75] - for an indication of what are the biological features relevant to the different morphologies. 


\subsection{Chemotactic-based branching growth: insights from the Communicating Walkers model}

Ben-Jacob et al. argued that for the colonial adaptive self-organization the $\mathscr{T}$ morphotype employs several kinds of chemotactic responses. Usually chemotactic response means a bias of the movement in response to a gradient of an externally produced field like in the case of food chemotaxis. However, it could also be a response to a field produced directly or indirectly by the bacterial cells - chemotactic signaling.

Three kinds of chemotactic responses are suggested to be employed by the $\mathscr{T}$ morphotype, each dominant in different regime of the morphology diagram. One response is the food chemotaxis we have mentioned earlier. According to the "receptor law", it is expected to be dominant for some range of nutrient levels (the corresponding levels of peptone are determined by the constant $K$ ). The two other kinds of chemotactic responses are signaling chemotaxis. One is long-range repulsive chemotaxis where the chemical is secreted by starved bacteria at the inner parts of the colony. The second signal is a short-range attractive chemotaxis where the chemical is secreted by bacteria at the colony's front, bacteria which are immersed in toxic waste products. The length scale of each signal is determined by the diffusion constant of the chemical agent and the rate of its spontaneous decomposition.

Amplification of diffusive instability due to nutrients chemotaxis: In non-living systems, the more ramified patterns (lower fractal dimension) are observed for lower growth velocity. Based on growth velocity as function of peptone level and based on growth dynamics, Ben-Jacob et al. concluded that in the case of bacterial colonies there is a need for mechanism that can both increase the growth velocity and maintain, or even decrease, the fractal dimension. Ben-Jacob et al. suggested food chemotaxis to be the required mechanism. It provides an outward drift to the cellular movements; thus, it should increase the rate of envelope propagation. At the same time, being a response to an external field it should also amplify the basic diffusion instability of the nutrients field. Hence, it can support faster growth velocity together with a ramified pattern of low fractal dimension.

The Communicating Walkers model was used to test the above hypothesis. The simulations showed that as expected, the inclusion of food chemotaxis in the model led to a considerable increase of the growth velocity without significant change in the fractal dimension of the pattern.

Repulsive chemotactic signaling: We focus now on the formation of the fine radial branching patterns at low peptone levels. From the study of non-living systems it is known that, in the same manner that an external diffusion field leads to the diffusion instability, an internal diffusion field will stabilize the growth. It is natural to assume that such a field is produced by some sort of chemotactic agent. To regulate the organization of the branches, it must be a long-range signal. To result in radial branches it must be a repulsive chemical produced by cells at the inner parts of the colony. The must probable candidates are the bacteria entering a pre-spore stage due to depletion of nutrient. This proposal has also been verified by simulations of the Communicating 
Walkers model. In the presence of repulsive chemotaxis the patterns become much denser with a smooth circular envelope, while the branches are thinner and radially oriented.

\subsection{Results for the continuous models}

We incorporate the effect of chemotaxis in the continuous models by introducing a chemotactic flux $\boldsymbol{J}_{\text {chem }}$, which is written (for the case of a chemorepellent and a linear diffusion) as [73]

$$
\boldsymbol{J}_{\text {chem }}=-b \chi(r) \nabla r,
$$

where $r(\mathbf{x}, t)$ is the concentration of chemorepellent and $\chi(r)$ is the chemotactic sensitivity to the repellent. In the case of a chemoattractant, e.g. a nutrient, the expression for the flux will have an opposite sign.

Recall that in the case of the "receptor law", the sensitivity $\chi(r)$ takes the form:

$$
\chi(r)=\frac{\chi_{0} K}{(K+r)^{2}} .
$$

Thus, we obtain the reaction diffusion chemotaxis equation:

$$
\frac{\partial b}{\partial t}=-\nabla(-D \nabla b-b \chi(r) \nabla r)+f(b) .
$$

In addition, one has to write an equation describing the diffusion and the production and decomposition of the chemorepellent. This is written as follows:

$$
\dot{r}=D_{r} \nabla^{2} r+\Gamma_{r} s-\Omega_{r} b r-\lambda_{r} r,
$$

where $D_{r}$ is the diffusion coefficient of the chemorepellent, $\Gamma_{r}$ is the emission rate of repellent by pre-spores, $\Omega_{r}$ is the decomposition rate of the repellent by active bacteria, and $\lambda_{r}$ is the rate of self decomposition of the repellent.

We have tested the effect of food chemotaxis and repulsive chemotaxis in several of the aforementioned reaction-diffusion models, and present here results for the Kitsunezaki nonlinear diffusion model and the Mimura et al. meta-stable model.

When treating the non-linear diffusion model, we must modify the expression for the chemotactic flux, in order to incorporate the density dependence of the bacterial movement. Thus, similarly to the diffusion coefficient, which is written as $D_{b}=D_{0} b^{k}$, we modify the chemotactic flux to become:

$$
\boldsymbol{J}_{\text {chem }}=\zeta(b) \chi(r) \nabla r,
$$

where $\zeta(b)=b \cdot b^{k}=b^{k+1}$ is the bacterial response to the chemotactic agent. For the linear diffusion case, $\zeta(b)$ degenerates again to $b$. 

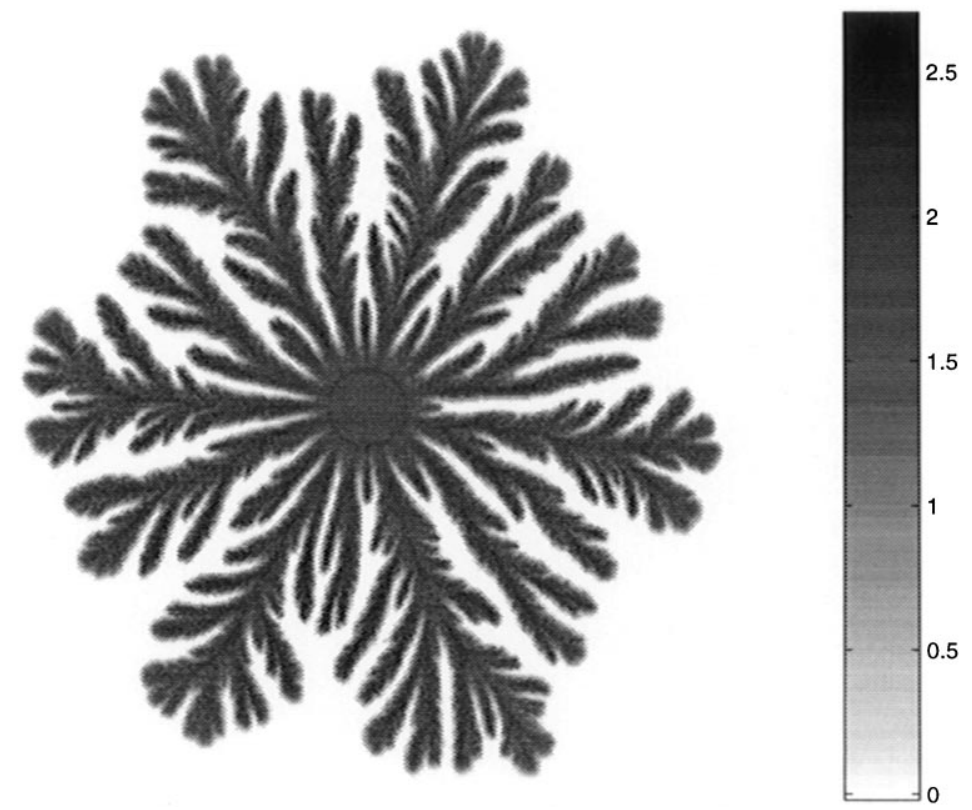

Fig. 27. 2D growth pattern $(b+s)$ of the Kitsunezaki model with food chemotaxis included. $\chi_{0 f}=3$. Other parameters as in Fig. 19.

Fig. 27 depicts a pattern developed by the Kitsunezaki model when food chemotaxis is included. All of the parameters are the same as in Fig. 19 (no chemotaxis). Although the patterns are very similar, the growth velocity when food chemotaxis is included is about twice the velocity in the absence of chemotactic response. In other words, the velocity is doubled with no change in the fractal dimension.

The effect of repulsive chemotactic signaling is demonstrated in Fig. 28 - again with otherwise identical parameters to those in Fig. 19. It can be seen that the previously fractal-like shape has turned into a radial branching pattern with a circular envelope.

Thus, for the two types of chemotactic response - food and repulsive, the results we observe are similar to those obtained for the communicating walkers model. This agreement is not surprising, as both models capture the important feature of a lubrication fluid. Recall also that the nonlinear diffusion model is the only one which exhibits a sharp front in $1 \mathrm{D}$.

The effect of chemotactic response in the Mimura et al. model is presented in Figs. 29 and 30. The chemotactic response was added to a previously DLA-like colony (Fig. 23a). The addition of food chemotaxis turns the colony into a densely branched one, with branches much thicker than before. The repulsive chemotaxis makes the branches radially oriented, but they become thicker than before. Thus, the effect of chemotactic responses in this model differs from the one obtained for both the walker model and the Kitsunezaki model. We believe this to stem from the fact that the model does not include nonlinear diffusion to capture the effect of lubrication. 

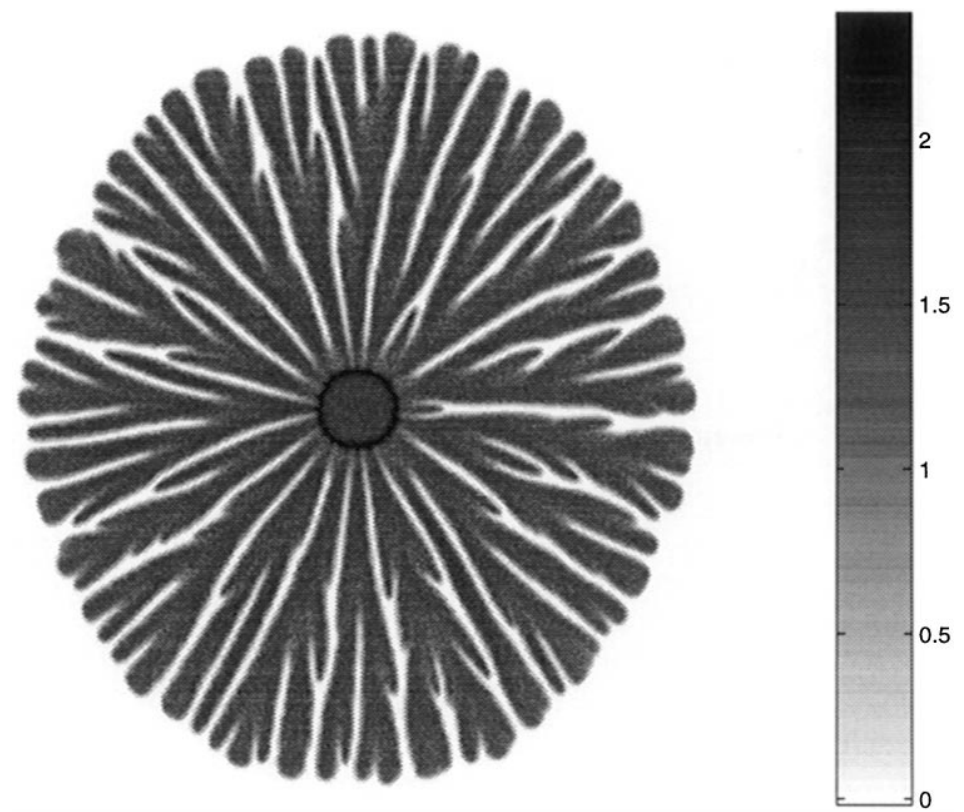

Fig. 28. 2D growth pattern $(b+s)$ of the Kitsunezaki model with repulsive chemotactic signaling included. Parameters are: $\chi_{0 r}=1, D_{r}=1, \Gamma_{r}=0.25, \Omega_{r}=0, \Lambda_{r}=0.001$. Other parameters as in Fig. 19.
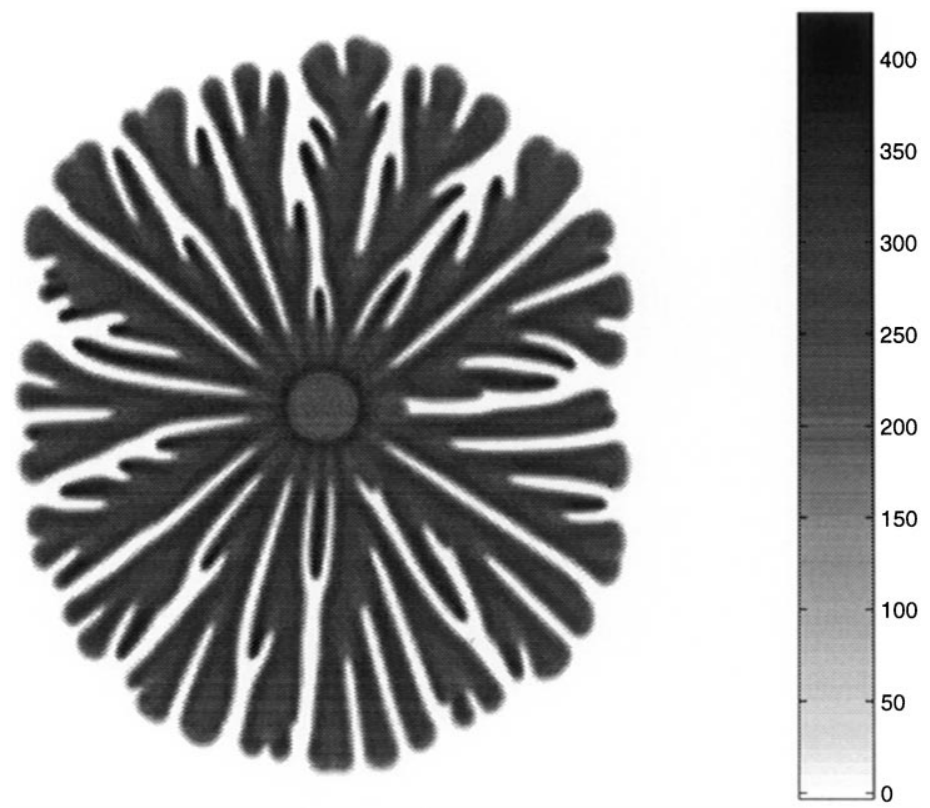

Fig. 29. 2D growth pattern $(b+s)$ of the Mimura et al. model with food chemotaxis included. $\chi_{0 f}=0.06$. Other parameters as in Fig. 23a. 

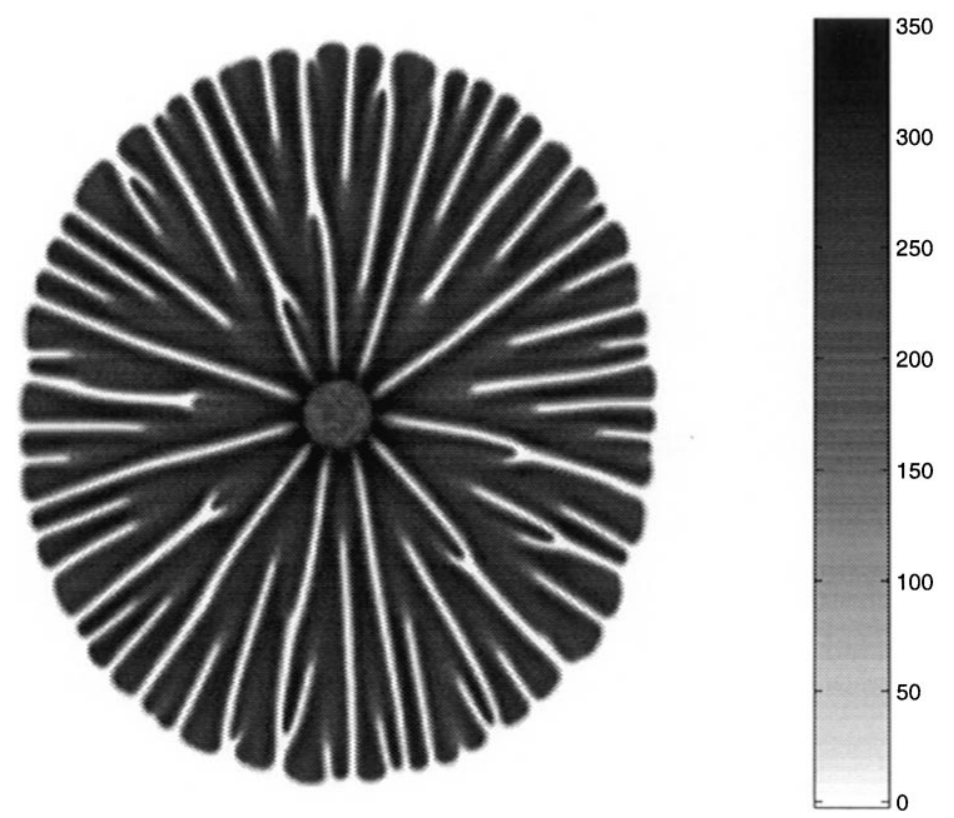

Fig. 30. 2D growth pattern $(b+s)$ of the Mimura et al. model with repulsive chemotactic signaling included. Parameters are: $\chi_{0 r}=0.1, D_{r}=1, \Gamma_{r}=0.2, \Omega_{r}=0, \Lambda_{r}=0.01$. Other parameters as in Fig. 23a

\section{Discussion}

We first briefly reviewed experimental observations of branching patterns in various bacterial strains, under a range of growth conditions. Both the colonial patterns and the optical microscope observations of the bacterial dynamics were presented. We have also included a brief summary of the known key biological features required, as we think, for successful modeling of the growth.

Our goal in this manuscript was to test reaction-diffusion models. To this end we surveyed the reaction-diffusion models for branching growth that we are familiar with. Mathematical analysis reveals that a number of different features can lead to instability of a propagating front: A density-dependent (i.e. nonlinear) diffusion coefficient; a lower cutoff in the growth term, and a meta-stable growth term. For this instability to create pronounced branches, a death term must be added to the growth. Such a term prevents bacteria inside the colony from moving and "healing" the dips on the surface. Making the diffusion term nutrient dependent can lead to a similar effect. The experimental observations provide a clear indication that the bacteria turn immobile, so that a "death" term indeed has to be included in the models.

The fact that different mathematical features can lead to similar (to the eye) branching patterns emphasizes how cautious we have to be in modeling the colonial development. True, it might be that different bacterial strains develop branching patterns by 
the employment of different biological features (each might correspond to one of the mathematical features). Yet, when we consider a specific bacterial strain, comparing the experimentally observed and model's patterns is not sufficient to tell us if indeed the right biological features are included in the model.

What, then, is the right way to tackle the problem? The integrative way: One must combine the mathematical knowledge (in this case, what mechanisms lead to instability and branching) with an attempt to model the (assumed) generic biological features, and comprehensive comparison with experimental results. As an example, the (mathematical) mechanism of non-linear diffusion leads to a branching colony, with compact support. We must then ask ourselves whether the movement of bacteria could be described in such a way. The answer is positive. As we have explained earlier, the lubrication fluid can be modeled by a nonlinear diffusion of the bacteria.

For more critique test of the models, additional aspects of the growth (such as functional dependence of the colonial growth velocity on growth conditions, branches size and width distributions, etc.) have to be compared with the model predictions. One should also compare the theory with more involved experimental tests, such as the effect of imposed anisotropy, competition between neighboring colonies, and expression of mutants (emergence of sectors) in expanding colonies.

Our conclusion from the study of bacterial branching growth is that the minimal features of diffusion, food consumption, reproduction and inactivation are not sufficient to explain the complete picture of the observed phenomena. We believe that additional mechanisms must be introduced, and propose chemotactic signaling as plausible one.

This work has dealt with continuous models. Such models are not preferable to discrete ones. Each has its advantages and disadvantages. The discrete walkers model, for example, enables us to include the valuable feature of internal degrees of freedom, but is computationally limited in the number of walkers that can be simulated, and thus its scaling to the real problem is somewhat problematic. The best strategy is to employ in parallel both the reaction-diffusion and the walkers approach.

To conclude, despite the difficulties and possible pitfalls, we hope to attract researchers to this emerging new endeavor. After all, a significant progress has been made towards working out the cybernetic processes (communication, regulation and control) during colonial development. Yet, many challenging puzzles are waiting to be solved and tantalizing phenomena are waiting to be discovered.

\section{Acknowledgements}

We have benefited from many discussions on the presented studies with H. Levine. We thank M. Matsushita for discussions about the Mimura et al. model. We are greatful to I. Rafols for sending us a copy of his master thesis which we found most helpful. IG wishes to thank R. Segev for fruitful discussions. Identifications of the $\mathscr{T}$ morphotype and genetic studies are carried out in collaboration with the group of D. Gutnick. Presented studies are supported in part by a grant from the Israeli Academy of Sciences 
grant no. 593/95 and by the Israeli-US Binational Science Foundation BSF grant no. 00410-95.

\section{Appendix A. From real units to dimensionless models}

Our goal is to relate the dimensionless equations with biophysical values of the parameters. The procedure is to set the dimensional units to be the natural scales.

\section{A.1. Deriving the equations}

The diffusive Fisher-Kolmogorov equations in dimensional form (Section 4.1) are

$$
\frac{\partial b}{\partial t}=D_{b} \nabla^{2} b+E_{b} n b, \quad \frac{\partial n}{\partial t}=D_{n} \nabla^{2} n-E_{n} n b,
$$

here $b$ and $n$ are the two dimensional concentration of bacteria and of nutrition, respectively. The field $b$ is measured in units of number of bacteria per area in $\mathrm{cm}^{2}$. The field $n$ is measured in units of grams per area in $\mathrm{cm}^{2}$. Experimentally nutrients are usually measured in $\mathrm{g} / 1$. Note that $1 \mathrm{~g} / 1$ corresponds to $0.3 \mathrm{mg} / \mathrm{cm}^{2} . D_{b}$ and $D_{n}$ are the corresponding diffusion coefficients in units of $\mathrm{cm}^{2} / \mathrm{s} . E_{b}$ is the bacterial reproduction rate in units of $\mathrm{s}^{-1}$ per nutrition concentration. $E_{n}$ is the nutrition consumption rate in units of $\mathrm{s}^{-1}$ per bacteria concentration.

We change the variables to be dimensionless:

$$
t \rightarrow t T, \quad x \rightarrow x X, \quad b \rightarrow b B, \quad n \rightarrow n N,
$$

the new variables are dimensionless and the capitals are their corresponding units. We let the temporal and spatial units be the natural scales. The (microscopic) time scale of the model is the bacterial reproduction time $\tau_{R}$, so $T=\tau_{R}$. The length scale is the diffusion length of the nutrition during reproduction time. The nutrition available for bacteria during reproduction time is proportional to the square of the diffusion length. The length unit is

$$
X=\sqrt{D_{n} \tau_{R}}
$$

After inserting the dimensionless variables into Eq. (A.1) we obtain

$$
\begin{aligned}
& \frac{\partial b}{\partial t}=D_{b} / D_{n} \nabla^{2} b+(T N) E_{b} b n, \\
& \frac{\partial n}{\partial t}=\nabla^{2} n-(T B) E_{n} b n .
\end{aligned}
$$


We define the relative diffusion coefficient $D \equiv D_{b} / D_{n}$ and impose the following relations:

$$
\begin{aligned}
& T B E_{n}=1, \\
& T N E_{b}=1 .
\end{aligned}
$$

We obtain the following dimensionless equations:

$$
\frac{\partial b}{\partial t}=D \nabla^{2} b+b n, \quad \frac{\partial n}{\partial t}=\nabla^{2} n-b n .
$$

\section{A.2. Evaluation of the parameters}

We will estimate the values of the parameters $E_{b}, E_{n}$ and $D_{n}$, and derive from them the dimensional units. A review of some of the following biological arguments appears in Section 3.4.

- The bacterial reproduction time, when bacteria grow under optimal conditions, is about $\tau_{R}=25 \mathrm{~min}$. Colonies which exhibit branching patterns grow under limited nutrition supply. Therefore the reproduction time will be longer, but in the same order of magnitude. We set the time unit to be

$$
T=\tau_{R}=25 \mathrm{~min} .
$$

- A typical value for the diffusion coefficient of chemicals in agar is $10^{-7} \mathrm{~cm}^{2} / \mathrm{s}$. So we assume that $D_{n}$, the diffusion coefficient of the nutrition in the agar, is similar. We can find the length unit using Eq. (A.3):

$$
X=0.01 \mathrm{~cm}=100 \mu \mathrm{m} .
$$

- The nutrition concentration in the experiments conducted by Ben-Jacob et al. [3], Rafols [60] and others was $0.1-5 \mathrm{mg} / \mathrm{cm}^{2}$. We set $N$ to have a similar value:

$$
N=1 \mathrm{mg} / \mathrm{cm}^{2}=10 \times 10^{-12} \mathrm{~g} / \mu^{2}=10^{-7} \mathrm{~g} / X^{2} .
$$

- The reproduction rate per bacterium (Eq. (A.1)) is $E_{b} N n$, where $n$ is the dimensionless concentration. The rate is the inverse of the reproduction time, which depends on the nutrition concentration. We assume that $N$ is the concentration for which the reproduction time is $\tau_{R}$. Therefore,

$$
E_{b} N=1 / \tau_{R}
$$

and Eq. (A.6) is satisfied.

- Similarly, $E_{n} N$ is the nutrition consumption rate per bacterium. We suggest that during reproduction time, a single bacterium consumes an amount of nutrition three times its mass, which is about $3 \times 10^{-12} \mathrm{~g}$. Therefore, the rate of nutrition 
consumption is

$$
E_{n} N \sim \frac{3 \times 10^{-12} \mathrm{~g} \text { nutrition }}{\text { bacteria }} \frac{1}{25 \mathrm{~min}} .
$$

From Eqs. (A.5) and (A.10) we obtain the bacterial concentration:

$$
B=N / T E_{n} N=3 \text { bacteria } / \mu^{2}=3 \times 10^{4} \text { bacteria } / X^{2} .
$$

The discrete time step of the numerical integration is measured in units of $T$. Sometimes numeric stability demands that the time step will be less than one. Then, as an example, a time step of 0.001 will correspond to $0.001 T \sim 1 \mathrm{~s}$.

\section{A.3. Equations with cutoff in the reaction term}

The scaling performed in the previous section is also suitable for the cutoff equations (Section 4.2). We are interested in the meaning of the cutoff $\beta$. Since $\beta$ is measured in units of B, we use Eq. (A.13) to translate numerical values to bacteria concentrations. For example, $\beta=3 \times 10^{-5}$ corresponds to 1 bacteria $/ X^{2}$, while $\beta=1$ corresponds approximately to bacteria covering the agar surface in a continuous layer.

\section{A.4. Mimura et al. model}

The model's equations in dimensional form (Section 4.5) are

$$
\begin{aligned}
& \frac{\partial b}{\partial t}=D_{b} \nabla^{2} b+E_{b} b n-\frac{a_{0} b}{\left(a_{n}+n\right)\left(a_{b}+b\right)}, \\
& \frac{\partial n}{\partial t}=D_{n} \nabla^{2} n-E_{n} b n, \\
& \frac{\partial s}{\partial t}=\frac{a_{0} b}{\left(a_{n}+n\right)\left(a_{b}+b\right)},
\end{aligned}
$$

where $a_{n}$ and $a_{b}$ are constants which have the same units as $n$ and $b$ respectively. We introduce dimensionless variables as previously (Eq. A.2), and impose relation (A.5). We obtain:

$$
\begin{aligned}
& \frac{\partial b}{\partial t}=D_{b} / D_{n} \nabla^{2} b+\frac{E_{b} N}{E_{n} B} b n-\frac{a_{0} T}{B N} \frac{b}{\left(a_{n} / N+n\right)\left(a_{b} / B+b\right)}, \\
& \frac{\partial n}{\partial t}=\nabla^{2} n-b n, \\
& \frac{\partial s}{\partial t}=\frac{a_{0} T}{B N} \frac{b}{\left(a_{n} / N+n\right)\left(a_{b} / B+b\right)} .
\end{aligned}
$$

We define new parameters:

$$
D \equiv D_{b} / D_{n}
$$




$$
\begin{gathered}
\varepsilon \equiv \frac{E_{b} N}{E_{n} B}, \\
\mu \equiv \frac{a_{0} T}{B N} .
\end{gathered}
$$

We set $a_{n} / N=1$ and $a_{b} / B=1$ in the last term of the bacterial equation. (The assignment is acceptable since Mimura et al. does not justify the exact form of that term, rather they state that it is only its general properties that matters.) Changing to the new parameters gives the dimensionless equations.

Scaling this model requires adjusting the values of the units. Relation (A.6) is replaced by relation (A.17), which is equivalent to

$$
T N E_{b}=\varepsilon .
$$

Since Eq. (A.11) is still valid, we leave $N$ unchanged. The other units re-scale according to

$$
T \rightarrow \varepsilon T, \quad X \rightarrow \sqrt{\varepsilon} X, \quad B \rightarrow B / \varepsilon
$$

compared to the values of the units evaluated in Section A.2.

\section{References}

[1] M. Doudoroff, R.Y. Stainer, E.A. Adelberg, The Microbial World, Prentice-Hall, Englewood Cliffs, NJ, 1957.

[2] J.A. Shapiro, Bacteria as multicellular organisms, Scientific American 258 (6) (1988) 62-69.

[3] E. Ben-Jacob, From snowflake formation to the growth of bacterial colonies. Part II: Cooperative formation of complex colonial patterns, Contemp. Phys. 38 (1997) 205-241.

[4] E. Ben-Jacob, I. Cohen, H. Levine, Cooperative self-organization of microorganisms, Adv. Phys. (1998), in press.

[5] H. Levine, E. Ben-Jacob, The art and science of microorganisms, Scientific American (1998), in press.

[6] N.H. Mendelson, Helical Bacillus subtilis macrofibers: morphogenesis of a bacterial multicellular macroorganism, Proc. Natl. Acad. Sci. USA 75 (5) (1978) 2478-2482.

[7] P. Devreotes, Dictyostelium discoideum: a model system for cell-cell interactions in development, Science 245 (1989) 1054-1058.

[8] T. Matsuyama, K. Kaneda, Y. Nakagawa, K. Isa, H. Hara-Hotta, I. Yano, A novel extracellular cyclic lipopeptide which promotes flagellum-dependent and -independent spreading growth of serratia marcescens, J. Bacteriol. 174 (1992) 1769-1776.

[9] R.M. Harshey, Bees aren't the only ones: swarming in gram-negative bacteria, Mol. Microbiol. 13 (1994) 389-394.

[10] W.C. Fuqua, S.C. Winans, E.P. Greenberg, Quorum sensing in bacteria: the LuxR-LuxI family of cell density-responsive transcriptional regulators, J. Bacteriol. 176 (1994) 269-275.

[11] A. Latifi, M.K. Winson, M. Foglino, B.W. Bycroft, G.S. Stewart, A.Lazdunski, P. Williams, Multiple homologues of luxr and luxi control expression of virulence determinants and secondary metabolites through quorum sensing in pseudomonas aeruginosa pao1, Mol. Microbiol. 17 (1995) 333-343

[12] C. Fuqua, S.C. Winans, E.P. Greenberg, Census and consensus in bacterial ecosystems: the LuxR-LuxI family of quorum-sensing transcriptional regulators, Annu. Rev. Microbiol. 50 (1996) 727-751.

[13] E.O. Budrene, H.C. Berg, Complex patterns formed by motile cells of esherichia coli, Nature 349 (1991) 630-633.

[14] Y. Blat, M. Eisenbach, Tar-dependent and -independent pattern formation by Salmonella typhimurium, J. Bacteriol. 177 (7) (1995) 1683-1691. 
[15] E.O. Budrene, H.C. Berg, Dynamics of formation of symmetrical patterns by chemotactic bacteria, Nature 376 (1995) 49-53.

[16] J.A. Shapiro, D. Trubatch, Sequential events in bacterial colony morphogenesis, Physica D 49 (1991) 214-223.

[17] B. Salhi, N.H. Mendelson, Patterns of gene expression in Bacillus subtilis colonies, J. Bacteriol. 175 (1993) 5000-5008.

[18] N.H. Mendelson, B. Salhi, Patterns of reporter gene expression in the phase diagram of Bacillus subtilis colony forms, J. Bacteriol. 178 (1996) 1980-1989.

[19] T. Galitski, J.R. Roth, Evidence that F plasmid transfer replication underlies apparent adaptive mutation, Science 268 (1995) 421-423.

[20] J.P. Rasicella, P.U. Park, M.S. Fox, Adaptive mutation in Escherichia coli: a role for conjugation, Science 268 (1995) 418-420.

[21] R.V. Miller, Bacterial gene swapping in nature, Scientific American (January 1998).

[22] J.O. Kessler, Co-operative and concentrative phenomena of swimming micro-organisms, Contemp. Phys. 26 (1985) 147-166.

[23] H. Fujikawa, M. Matsushita, Fractal growth of Bacillus subtilis on agar plates, J. Phys. Soc. Japan 58 (1989) 3875-3878.

[24] T.J. Pedley, J.O. Kessler, Bioconvection, Sci. Prog. 76 (1989) 105-123.

[25] E. Ben-Jacob, H. Shmueli, O. Shochet, A. Tenenbaum, Adaptive self-organization during growth of bacterial colonies, Physica A 187 (1992) 378-424.

[26] T. Matsuyama, R.M. Harshey, M. Matsushita, Self-similar colony morphogenesis by bacteria as the experimental model of fractal growth by a cell population, Fractals 1 (3) (1993) 302-311.

[27] E. Ben-Jacob, O. Shochet, A. Tenenbaum, I. Cohen, A. Czirók, T. Vicsek, Generic modeling of cooperative growth patterns in bacterial colonies, Nature 368 (1994) 46-49.

[28] E. Ben-Jacob, I. Cohen, O. Shochet, I. Aronson, H. Levine, L. Tsimering, Complex bacterial patterns, Nature 373 (1995) 566-567.

[29] D.E. Woodward, R. Tyson, M.R. Myerscough, J.D. Murray, E.O. Budrene, H.C. Berg, Spatio-temporal patterns generated by salmonella typhimurium, Biophys. J. 68 (1995) 2181-2189.

[30] E. Ben-Jacob, I. Cohen, A. Czirók, T. Vicsek, D.L. Gutnick, Chemomodulation of cellular movement and collective formation of vortices by swarming bacteria and colonial development, Physica A 238 (1997) 181-197.

[31] J.O. Kessler, M.F. Wojciechowski, Collective behavior and dynamics of swimming bacteria, in: J.A. Shapiro, M. Dworkin (Eds.), Bacteria as Multicellular Organisms, Oxford University Press, New York, 1997, pp. 417-450.

[32] S.E. Esipov, J.A. Shapiro, Kinetic model of proteus mirabilis swarm colony development, J. Math. Biol. 36 (1998) 249-268.

[33] M. Matsushita, H. Fujikawa, Diffusion-limited growth in bacterial colony formation, Physica A 168 (1990) 498-506.

[34] H. Fujikawa, M. Matsushita, Bacterial fractal growth in the concentration field of nutrient, J. Phys. Soc. Japan 60 (1991) 88-94.

[35] R.N. Smith, F.E. Clarck, Motile colonies of bacillus alvei and other bacteria, J. Bacteriol. 35 (1938) 59-60.

[36] T.H. Henrici, The Biology of Bacteria: The Bacillaceae, 3rd ed., Heath, Lexington, MA, 1948.

[37] T.A. Witten, L.M. Sander, Diffusion-limited aggregation, a kinetic critical phenomenon, Phys. Rev. Lett. 47 (1981) 1400.

[38] L.M. Sander, Fractal growth processes, Nature 322 (1986) 789-793.

[39] T. Vicsek, Fractal Growth Phenomena, World Scientific, New York, 1989.

[40] T. Matsuyama, M. Matsushita, Morphogenesis by bacterial cells, in: P.M. Iannaccone, M.K. Khokha, (Eds.), Fractal Geometry in Biological Systems, an Analytical Approach, CRC Press, New York, 1995, pp. $127-171$.

[41] E. Ben-Jacob, A. Tenenbaum, O. Shochet, O. Avidan, Holotransformations of bacterial colonies and genome cybernetics, Physica A 202 (1994) 1-47.

[42] M. Tcherpikov, E. Ben-Jacob, D. Gutnick, Identification of two pattern-forming strains and their localization in a phylogenetic cluster, Int. J. Syst. Bacteriol. (1998), in press.

[43] E. Ben-Jacob, I. Cohen, D. Gutnick, Cooperative organization of bacterial colonies: From genotype to morphotype, Annu. Rev. Microbiol. (1998), in press. 
[44] T. Matsuyama, M. Matsushita, Fractal morphogenesis by a bacterial cell population, Crit. Rev. Microbiol. 19 (1993) 117-135.

[45] D.A. Kessler, J. Koplik, H. Levine, Pattern selection in fingered growth phenomena, Adv. Phys. 37 (1988) 255.

[46] J.S. Langer, Dendrites, viscous fingering, and the theory of pattern formation, Science 243 (1989) $1150-1154$.

[47] E. Ben-Jacob, P. Garik, The formation of patterns in non-equilibrium growth, Nature 343 (1990) 523-530.

[48] E. Ben-Jacob, From snowflake formation to the growth of bacterial colonies. Part I: Diffusive patterning in non-living systems, Contemp. Phys. 34 (1993) 247-273.

[49] J. Horgan, From complexity to perplexity, Scientific American 95 (1995) 74-79.

[50] D.A. Kessler, H. Levine, Pattern formation in dictyostelium via the dynamics of cooperative biological entities, Phys. Rev. E 48 (1993) 4801-4804.

[51] M.Y. Azbel, Survival-extinction transition in bacteria growth, Europhys. Lett. 22 (4) (1993) 311-316.

[52] E. Ben-Jacob, I. Cohen, O. Shochet, A. Czirók, T. Vicsek, Cooperative formation of chiral patterns during growth of bacterial colonies, Phys. Rev. Lett. 75 (15) (1995) 2899-2902.

[53] D.A. Kessler, H. Levine, L. Tsimring, Computational modeling of mound development in dictyostelium, Physica D 106 (3-4) (1997) 375-388.

[54] H. Parnas, L. Segel, A computer simulation of pulsatile aggregation in Dictyostelium discoideum, J. Theor. Biol. 71 (1978) 185-207.

[55] S.A. Mackay, Computer simulation of aggregation in dictyostelium discoideum, J. Cell. Sci. 33 (1978) $1-16$.

[56] M. Mimura, H. Sakaguchi, M. Matsushita, A reaction-diffusion approach to bacterial colony formation, Preprint, 1997.

[57] M. Matsushita, J. Wakita, H. Itoh, I. Rafols, T. Matsuyama, H. Sakaguchi, M. Mimura, Interface growth and pattern formation in bacterial colonies, Physica A 249 (1998) 517-524.

[58] K. Kawasaki, A. Mochizuki, M. Matsushita, T. Umeda, N. Shigesada, Modeling spatio-temporal patterns created by bacillus-subtilis, J. Theor. Biol. 188 (1997) 177-185.

[59] S. Kitsunezaki, Interface dynamics for bacterial colony formation, J. Phys. Soc. Japan 66 (5) (1997) $1544-1550$.

[60] I. Rafols, Formation of concentric rings in bacterial colonies, MSc thesis, Chuo University, Japan, 1998.

[61] D.A. Kessler, H. Levine, Fluctuation-induced diffusive instabilities, Nature 394 (1998) 556-558.

[62] E. Ben-Jacob, O. Shochet, A. Tenenbaum, I. Cohen, A. Czirók, T. Vicsek, Communication, regulation and control during complex patterning of bacterial colonies, Fractals 2 (1) (1994) 15-44.

[63] E. Ben-Jacob, P. Garik, T. Muller, D. Grier, Characterization of morphology transitions in diffusioncontrolled systems, Phys. Rev. A 38 (1988) 1370.

[64] M. Eisenbach, Functions of the flagellar modes of rotation in bacterial motility and chemotaxis, Mol. Microbiol. 4 (2) (1990) 161-167.

[65] J. Henrichsen, Bacterial surface translocation: a survey and a classification, Bac. Rev. 36 (1972) $478-503$.

[66] H.C. Berg, Random Walks in Biology, Princeton University Press, Princeton, NJ, 1993.

[67] J. Adler, Chemoreceptors in bacteria, Science 166 (1969) 1588-1597.

[68] H.C. Berg, E.M. Purcell, Physics of chemoreception, Biophys. J. 20 (1977) 193-219.

[69] J.M. Lackiie (Ed.), Biology of the Chemotactic Response, Cambridge University Press, Cambridge, 1986.

[70] D.B. Dusenbery, Spatial sensing of stimulus gradients can be superior to temporal sensing for freeswimming bacteria, Biophys. J. 74 (1998) 2272-2277.

[71] J. Adler, Chemotaxis in bacteria, Science 153 (1966) 708-716.

[72] H.C. Berg, D.A. Brown, Chemotaxis in Escherichia coli analyzed by three-dimensional tracking, Nature 239 (1972) 500-504.

[73] J.D. Murray, Mathematical Biology, Springer, Berlin, 1989.

[74] A.J. Wolfe, H.C. Berg, Migration of bacteria in semisolid agar, Proc. Natl. Acad. Sci. USA 86 (1989) 6973-6977.

[75] I. Cohen, A. Czirók, E. Ben-Jacob, Chemotactic-based adaptive self organization during colonial development, Physica A 233 (1996) 678-698.

[76] R.A. Fisher, The wave of advance of advantageous genes, Annu. Eugenics 7 (1937) 255-369. 
[77] A.I. Kolmogorov, I. Petrovsky, N. Piscounov, Étude de l'équation de la diffusion avec croissance de la quantité de matière et son application à un problème biologique, Moscow Univ. Bull. Math. 1 (1937) $1-25$.

[78] D.G. Aronson, H.F. Weinberger, Nonlinear diffusion in population genetics, combustion, and nerve pulse propagation. in: Partial Differential Equations and Related Topics, Lecture Notes in Mathematics, Springer, Berlin, 1975, pp. 5-49.

[79] E. Ben-Jacob, H. Brand, G. Dee, L. Kramer, J.S. Langer, Pattern propagation in nonlinear dissipative systems, Physica D 14 (1985) 348-364.

[80] W. van Saarloos, Front propagation into unstable states: marginal stability as a dynamical mechanism for velocity selection, Phys. Rev. A 37 (1988) 211-229.

[81] W. van Saarloos, Front propagation into unstable states: II. Linear versus non-linear marginal stability and rate of convergence, Phys. Rev. A 39 (1989) 6367-6389.

[82] M. Cross, P. Hohenberg, Rev. Mod. Phys. 65 (1993) 851.

[83] D.A. Kessler, Z. Ner, L.M. Sander, Front propagation: precursors, cutoffs and structural stability, Preprint, 1998.

[84] W.W. Mullins, R.F. Sekerka, Stability of a planar interface during solidification of a dilute binary alloy, J. Appl. Phys. 35 (1964) 444.

[85] M. Matsushita, J.-I. Wakita, T. Matsuyama, Growth and morphological changes of bacteria colonies, in: P.E. Cladis, P. Palffy-Muhoray (Eds.), Spatio-Temporal Patterns in Nonequilibrium Complex Systems, Santa-Fe Institute Studies in the Sciences of Complexity, Addison-Wesley, Reading, MA, 1995, pp. 609-618.

[86] E. Ben-Jacob, O. Shochet, A. Tenenbaum, O. Avidan, Evolution of complexity during growth of bacterial colonies, in: P.E. Cladis, P. Palffy-Muhoray (Eds.), Spatio-Temporal Patterns in Nonequilibrium Complex Systems, Santa-Fe Institute Studies in the Sciences of Complexity, Addison-Wesley, Reading, MA, 1995, pp. 619-634.

[87] J. Wakita, K. Komatsu, A. Nakahara, T. Matsuyama et al., Experimental investigation on the validity of population dynamics approach to bacterial colony formation, J. Phys. Soc. Japan 63 (1994) 1205-1211.

[88] R. Kupferman, O. Shochet, E. Ben-Jacob, Numerical study of morphology diagram in the large undercooling limit using a phase-field model, Phys. Rev. E 50 (1994) 1005.

[89] R. Kupferman, Morphology, coexistence and selection in interfacial pattern formation, $\mathrm{PhD}$ thesis, Tel-Aviv University, 1995.

[90] I. Cohen, Mathematical Modeling and Analysis of Pattern Formation and Colonial Organization in Bacterial Colonies, MSc thesis, Tel-Aviv University, Israel, 1997.

[91] E. Ben-Jacob, H. Levine, I. Cohen, Cooperative self-organization of microorganisms, Adv. Phys., to appear.

[92] W.I. Newman, The long-time behavior of the solution to a non-linear diffusion problem in population genetics and combustion, J. Theor. Biol. 104 (1983) 473-484.

[93] Y. Tu, H. Levine, D. Ridgway, Morphology transition in a mean-field model of diffusion-limited growth, Phys. Rev. Lett. 71 (23) (1993) 3838-3841.

[94] E. Ben-Jacob, O. Shochet, I. Cohen, A. Tenenbaum, A. Czirók, T. Vicsek, Cooperative strategies in formation of complex bacterial patterns, Fractals 3 (1995) 849-868. 